\title{
Lipopolysaccharide induces the differentiation of hepatic progenitor cells into myofibroblasts constitutes the hepatocarcinogenesis- associated microenvironment
}

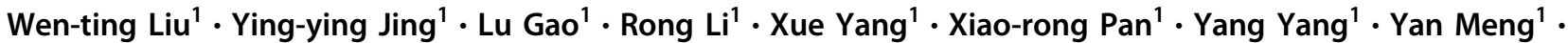

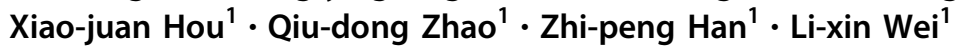

Received: 1 August 2018 / Revised: 5 April 2019 / Accepted: 16 April 2019 / Published online: 7 May 2019

(c) ADMC Associazione Differenziamento e Morte Cellulare 2019

\begin{abstract}
Hepatocellular carcinoma (HCC) generally occurs in the presence of chronic liver injury, often as a sequela of liver fibrosis. Hepatic progenitor cells (HPCs) are known to be capable of forming both hepatocytes and cholangiocytes in chronic liver injury, which are also considered a source of myofibroblasts and tumor-initiating cells, under carcinogenic circumstances. However, the underlying mechanisms that activate HPCs to give rise to HCC are still unclear. In current study, the correlation between HPCs activation and liver fibrosis and carcinogenesis was investigated in rats and human specimens. We analyzed the role of HPCs in tumorigenesis, by transplanting exogenous HPCs in a diethylnitrosamine-induced rat HCC model. Our data indicated that HPC activation correlated with hepatic fibrosis and hepatocarcinogenesis. We further found that exogenous HPC infusion promoted liver fibrosis and hepatocarcinogenesis, while lipopolysaccharides (LPS) played an important role in this process. However, results of our study indicated that LPS did not induce HPCs to form tumor in nude mice directly. Rather, LPS induced myofibroblast-like morphology in HPCs, which enhanced the tumorigenic potential of HPCs. Further experiments showed that LPS/Toll-like receptor 4 (TLR4) signaling mediated the differentiation of HPCs into myofibroblasts and enhanced the production of interleukin-6 (IL-6) and tumor necrosis factor- $\alpha$ (TNF- $\alpha$ ), which led to the aberrant expression of Ras and p53 signaling pathways in HPCs, and finally, promoted the proliferation and malignant transformation of HPCs, by long non-coding RNA regulation. Besides, examination of HCC clinical samples demonstrated that IL- 6 and TNF- $\alpha$ production correlated with HPC activation, hepatic fibrosis, and HCC recurrence. Our study indicates that both myofibroblasts and tumor cells are derived from HPCs. HPC-derived myofibroblasts create tumor microenvironment and contribute to the proliferation and malignant transformation of HPCs. Furthermore, LPS present in the chronic liver inflammation microenvironment might play an important role in hepatocarcinogenesis, by regulating the plastic potential of HPCs.
\end{abstract}

These authors contributed equally: Wen-ting Liu, Ying-ying Jing, Lu Gao

Edited by M Piacentini

Supplementary information The online version of this article (https:// doi.org/10.1038/s41418-019-0340-7) contains supplementary material, which is available to authorized users.

Zhi-peng Han

hanzhipeng0311@126.com

$\triangle$ Li-xin Wei

weilixin_smmu@163.com

1 Tumor Immunology and Gene Therapy Center, Shanghai Eastern Hepatobiliary Surgery Hospital, Shanghai, China
Hepatocellular carcinoma (HCC) is the fifth most commonly diagnosed cancer and the third cause of cancer death worldwide [1]. The development of HCC is associated with chronic liver inflammation, fibrosis, and its subsequent irreversible cirrhosis [2]. Hepatic fibrosis is a pathological consequence of chronic liver diseases, and results from the progressive accumulation of altered extracellular matrix, highly enriched in type I and III fibrillar collagens; [3] in advanced stages, fibrosis leads to cirrhosis. Clinical consequences of cirrhosis are: failure in the synthetic function of the liver, portal hypertension, high susceptibility to infection, and high risk to develop HCC [3]. While the histopathologic features of HCC are well established, the molecular mechanisms of the cancer-promoting effectors, including fibrosis, are not well understood. 
Hepatic progenitor cells (HPCs), which are denoted as oval cells in rodents, reside in the smallest and most peripheral branches of the biliary tree, ductules, and canals of Hering [4-6]. Results of numerous studies indicate that HPCs can differentiate toward the hepatocytic and the biliary lineage [7]. HPC activations are closely related to liver fibrosis [8, 9]. Several studies have shown that activated HPCs release cytokines to activate quiescent hepatic stellate cells to promote liver fibrosis [10]. In addition, a recent study has demonstrated that myofibroblasts are generated from HPCs [11]. In our previous study, we found that lipopolysaccharide (LPS) could effectively promote the differentiation of HPCs into myofibroblasts [12]. Injured liver affects the permeability of the gut, which facilitates the abnormal translocation of bacteria and their metabolites, such as LPS, across the gastrointestinal barrier, into the portal circulation. LPS can induce the production of inflammatory cytokines, including interleukin-1 $\beta$ (IL-1 $\beta$ ), IL-6, type I interferons, transforming growth factor-beta (TGF- $\beta$ ), and tumor necrosis factor- $\alpha$ (TNF- $\alpha$ ) [13, 14]. Recent reports have demonstrated that LPS played an important role in liver damage and development of HCC [15-17]. The results of our study also reported that LPS could promote the proliferation of mouse-derived HPCs in vitro and in vivo. Moreover, LPS blocked the hepatic and biliary differentiation of multipotent HPCs (mHPCs) [18].

There is extensive evidence to indicate that $\mathrm{HCC}$ is a stem cell-derived disease. In addition, human HPCs have been discerned adjacent to HCC in hepatitis B virusinduced cirrhosis $[19,20]$, which is considered to represent precursor lesions of HCC. These results hint that HPCs may play a role in liver tumor development. However, the mechanisms by which HPCs give rise to $\mathrm{HCC}$ are still unclear, and whether genetic alterations play a role in this event is unknown. Therefore, the aim of the present study was to investigate the evolution of HPCs in tumor microenvironment and the potential mechanism underlying the process of hepatocarcinogenesis.

\section{Results}

\section{HPC activation correlated to liver fibrosis and hepatocarcinogenesis in primary HCC model and clinical HCC specimens}

In the diethylnitrosamine (DEN)-induced primary rat $\mathrm{HCC}$ model, we found that hepatocarcinogenesis was accompanied by the development of hepatic fibrosis (Fig. 1a). The expression of $\alpha$-smooth muscle actin ( $\alpha$-SMA) was increased during hepatocarcinogenesis (Fig. 1b). Consistent with the data, HPC activation (OV6 expression) was also enhanced during HCC occurrence (Fig. 1c). Both $\alpha$-SMA and OV6 expression was associated with HCC occurrence $(r=0.8, p<0.001 ; r=0.784, p<0.001)$ (Fig. 1d, e), and $\alpha-$ SMA expression correlated with OV6 expression $(r=0.658$, $p<0.001$ ) (Fig. 1f). The results indicate that HPC activation plays an important role in liver fibrosis and carcinogenesis.

Then, the association between HPC activation, liver fibrosis, and HCC occurrence was analyzed in clinical HCC specimens. According to the peritumoral immunohistochemistry results, all $84 \mathrm{HCC}$ patients were divided into the following groups: $\alpha$-SMA high expression group $(n=$ 42) and low expression group $(n=42)$, and OV6 high expression group $(n=40)$ and low expression group $(n=$ 44) (Fig. 1g, h). We further examined the correlation between OV6 and $\alpha$-SMA expression by comparing the integrated optical density (IOD) from image analysis. OV6 had a positive correlation with $\alpha$-SMA $(r=0.297, p<0.01)$ in the entire group (Fig. 1i). As shown in Fig. $1 \mathrm{j}$, patients with high OV6 expression were likely to be having significantly earlier recurrence than patients with low OV6 expression $(p<0.001)$. K7 expression was also detected as HPC expansion, and the same results were obtained (Supplementary Fig. 1). Additionally, $\alpha$-SMA high expression group also showed higher recurrence rate than low expression group $(p<0.05$, Fig. $1 \mathrm{k})$. These results suggest that HPC activation correlates to hepatic fibrosis and HCC recurrence.

\section{HPC transplantation promoted hepatocarcinogenesis and liver fibrosis in primary rat HCC model}

We demonstrated that long-term exposure of mHPCs to LPS did not lead to a higher incidence of HCC in a primary mouse liver cancer model (Supplementary Fig. 2). We speculated that the liver of mouse could not form typical hepatic cirrhosis, which was different from the process of hepatocarcinogenesis of human being. We employed DENinduced primary rat $\mathrm{HCC}$ model to identify the role of HPCs in tumorigenesis. HPC cell line, WB-F344 cells, was intrasplenically (i.s.) injected into F344 rats during hepatocarcinogenesis (Fig. 2a). Transplantation of WB-F344 cells led to poorer survival and could effectively promote tumorigenesis in DEN-exposed rats (Fig. 2b-d). Besides, levels of alanine aminotransferase (ALT) and aspartate aminotransferase (AST) in WB-F344 cell transplantation group were obviously higher than those in the control group (Fig. 2e, f). Thereafter, the results from hematoxylin and eosin (H\&E) staining and Bio-Plex assay showed that HPC infusion exacerbated inflammatory response in the livers (Fig. 2g, Supplementary Fig. 3a). The results of Sirius Red staining and Masson's Trichrome staining showed that compared with the control group, the liver from the WBF344 infusion group demonstrated higher levels of fibrosis. 

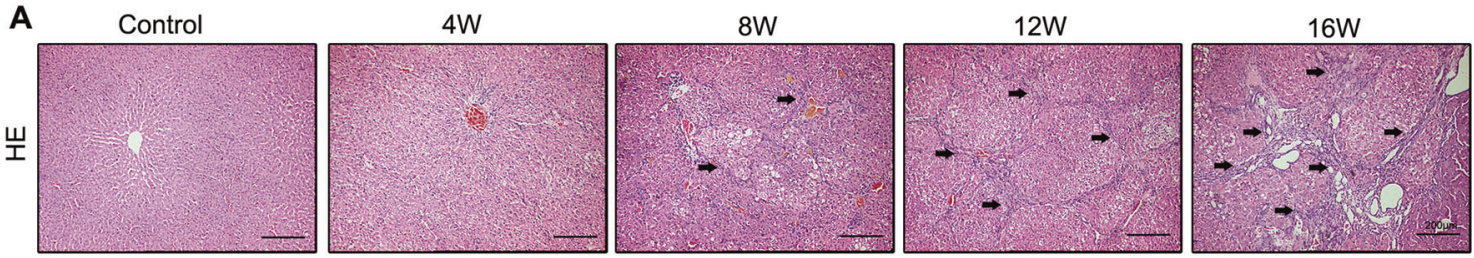

B Control

$4 \mathrm{~W}$

$8 W$
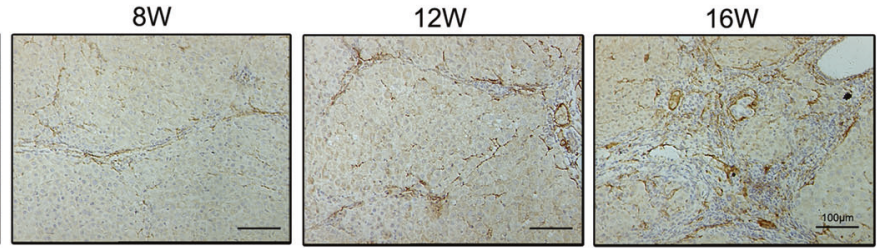

C

$4 \mathrm{~W}$

$8 W$

$12 \mathrm{~W}$
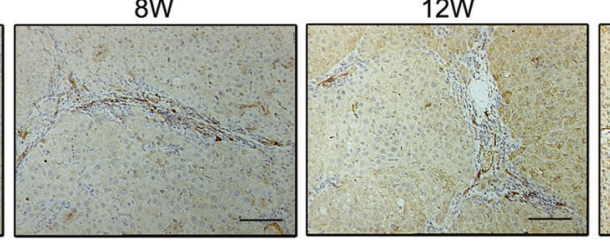

$16 \mathrm{~W}$
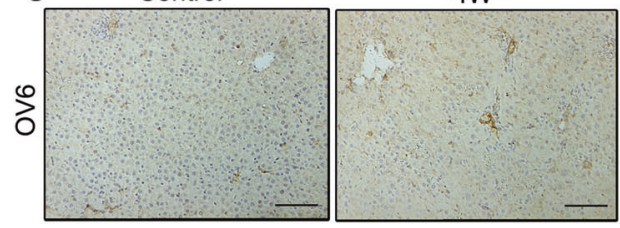

E $\widetilde{\S}^{0.4}$
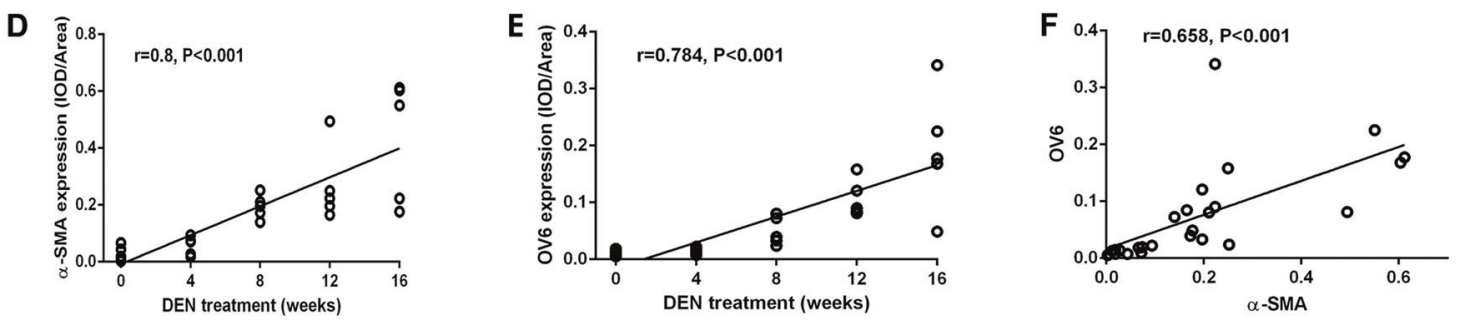

H

G
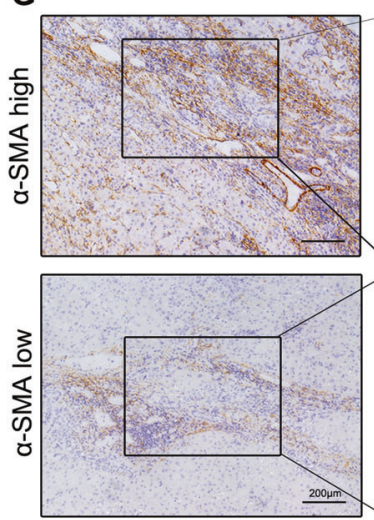

I

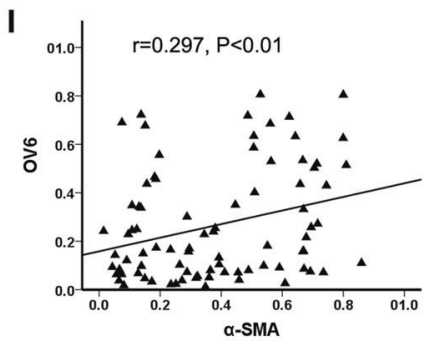

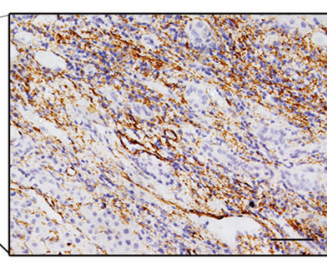

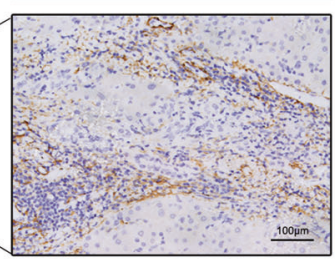

J

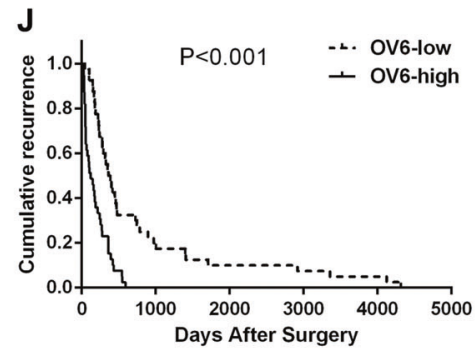

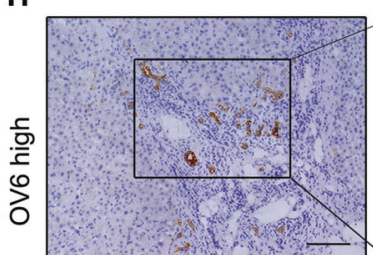
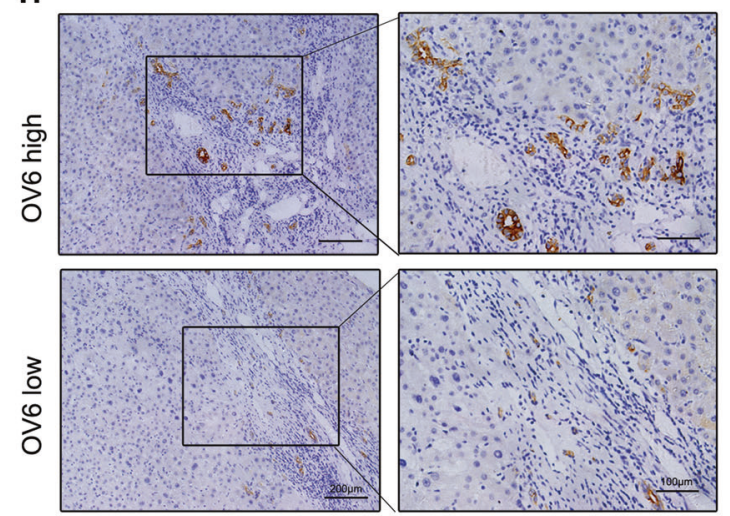

K

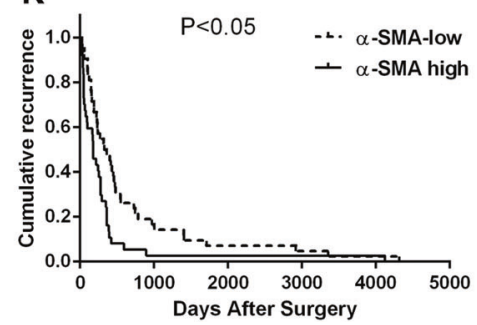

In addition, immunohistochemical (IHC) staining also showed that the WB-F344 group illustrated higher levels of $\alpha$-SMA expression (Fig. 2h). These results indicate that
HPC transplantation may promote hepatic fibrosis in DENinduced primary HCC model. Furthermore, we marked the WB-F344 cells with green fluorescent protein (GFP), by 
Fig. 1 Hepatic progenitor cell (HPC) activation correlated to liver fibrosis and hepatocarcinogenesis in primary rat hepatocellular carcinoma (HCC) model and clinical HCC specimens. a Hematoxylin and eosin (H\&E) staining was performed to observe the histological structure at different time points of diethylnitrosamine (DEN)-induced HCC rats, arrows mean the fibrosis structure in the liver section. b, $\mathbf{c}$ The expression of $\alpha$-smooth muscle actin ( $\alpha$-SMA) and OV6 was detected by immunohistochemical analysis. d, e The expression of $\alpha$ SMA and OV6 was evaluated at different time points in DEN-induced HCC rats; the data were measured by integrated optical density (IOD). Pearson's correlation analysis provided correlation coefficient $(r)$ and $p$ value. f Scatterplot with fitting line showed that the expression of OV6 strongly correlated with $\alpha$-SMA expression measured by IOD. Pearson's correlation analysis provided correlation coefficient $(r)$ and $p$ value. $\mathbf{g}, \mathbf{h} \alpha$-SMA and OV6 expression was detected in HCC peritumoral tissues by immunohistochemical analysis. i Scatterplot with fitting line showed that the expression of OV6 strongly correlated with $\alpha$-SMA expression measured by IOD. Pearson's correlation analysis provided correlation coefficient $(r)$ and $p$ value. $\mathbf{j}, \mathbf{k}$ Recurrence rate was analyzed in each group of HCC patients (Kaplan-Meier method), $p$ values were obtained by log-rank multiple comparison tests.

transfection with GFP lentiviral vector, to trace the evolution of WB-F344 cells in vivo. As shown in Fig. 2i, the presence of GFP-positive cells in the liver, after intrasplenic transplantation, indicated that WB-F344 cells had migrated and colonized in the liver. Immunofluorescence staining of $\alpha$-SMA, merged with GFP, indicated that WB-F344 cells might contribute to the formation of hepatic fibrosis, by differentiating into myofibroblasts.

In order to further confirm the role of HPCs on HCC incidence and hepatic fibrosis, we isolated primary HPCs from 2-acetylaminofluorine (2-AAF)/partial hepatectomy $(\mathrm{PH})$ rat model and the primary HPCs were transplanted into DEN-treated rats (Fig. 3a). As shown in Fig. 3b, c, transplantation of primary HPCs effectively enhanced the tumor incidence in DEN-induced HCC model. The level of ALT and AST also increased in HPC infusion group (Fig. 3d, e). H\&E staining demonstrated that primary HPC transplantation significantly lead to enhancement of inflammatory response in the liver tissues (Fig. 3f). Besides that, consistent with the effect of WB-F344 cells, the primary HPCs could also promote the hepatic fibrosis in DENexposed rats and lead to the upregulation of $\alpha$-SMA in liver tissues (Fig. 3g-i). These results confirm the effect of HPCs on hepatic fibrosis and hepatocarcinogenesis.

\section{LPS elimination alleviated HPC transplantation- induced liver fibrosis and hepatocarcinogenesis in primary HCC model}

LPS is a key pro-inflammatory cytokine in liver tumor microenvironment and plays an important role in liver damage and HCC [21]. We collected the serum from the portal vein at different times during the process of HCC model. The level of LPS increased during the process of
DEN-induced hepatocarcinogenesis (Fig. 4a), which indicated that LPS might be a key factor in HCC-associated microenvironment. In order to identify the role of LPS in liver fibrosis and hepatocarcinogenesis induced by HPCs, a combination of four different antibiotics was used to eliminate gut-derived LPS during HCC occurrence. The results demonstrated that this combination of antibiotics could effectively reduce the LPS level in the serum during DEN treatment (Fig. 4a). We further observed the effect of HPC infusion on tumorigenesis, with or without LPS treatment. As shown in Fig. 4b, c, compared to the control group, LPS elimination significantly reduced the enhancement of tumorigenesis induced by HPCs. Moreover, survival rate analysis also showed that LPS elimination significantly increased survival in rats that were transplanted with WBF344 cells, during DEN treatment (Fig. 4d). Meanwhile, ALT and AST levels decreased in the LPS elimination group (Fig. 4e, f). The results from H\&E staining and BioPlex assay showed that LPS elimination could reduce inflammatory response that increased by HPCs (Fig. 4g, Supplementary Fig. 3b). Additionally, LPS elimination reversed the ability of HPCs to enhance hepatic fibrosis (Fig. $4 \mathrm{~h}-\mathrm{j}$ ). These results suggest that LPS plays an important role in controlling the ability of HPCs to augment hepatocarcinogenesis and liver fibrosis.

\section{LPS induced HPC differentiation into myofibroblasts, which enhanced tumorigenic potential of HPCs}

In order to clarify the role of LPS in HPC-mediated hepatocarcinogenesis, we treated WB-F344 cells with LPS $(10 \mu \mathrm{g} / \mathrm{ml})$ for 2 weeks. As shown in Fig. 5a, the morphology of WB-F344 cells exhibited myofibroblast-like phenotype. LPS-pretreated WB-F344 cells were subcutaneously injected in BALB/c nu/nu mice. However, after 8 weeks of observation, we found that LPS pretreatment did not increase the tumorigenic potential in WB-F344 cells. Surprisingly, once LPS-pretreated WB-F344 cells and normal WB-F344 cells were mixed together and simultaneously injected subcutaneously, tumor nodules emerged (Fig. 5b, c). The histological type of these tumor nodules was confirmed as liver cancer; both $\alpha$-fetoprotein (AFP) and $\alpha$-SMA expression were detected in the tumor (Fig. 5d). These results indicate that LPS may induce the differentiation of HPCs into myofibroblasts, and that HPC-derived myofibroblasts enhance the tumorigenic potential of HPCs.

Furthermore, the impact of LPS on gene expression of WB-F344 cells was explored by transcriptomes analysis. We observed that 1590 genes were differentially expressed in the LPS-treated WB-F344 cells, including 588 upregulated genes and 1002 downregulated genes (Supplementary Fig. 4a). Further analysis showed that LPS treatment did not 
A

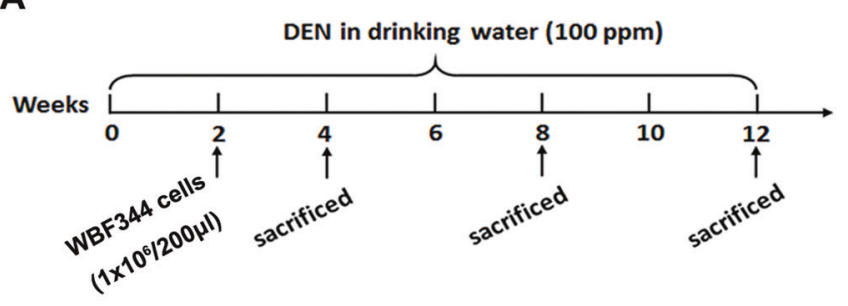

C
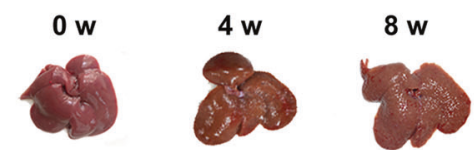

WBF344 cells
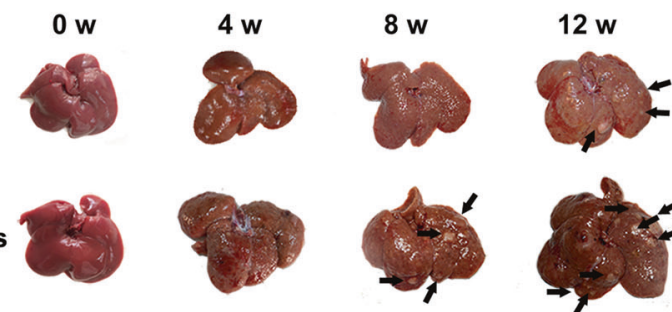

E

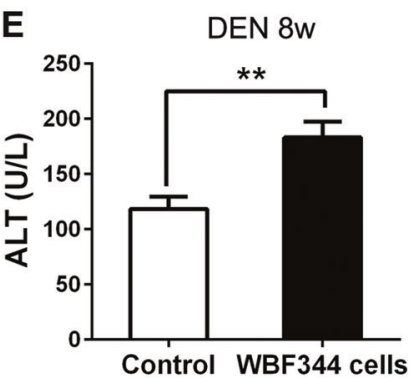

F

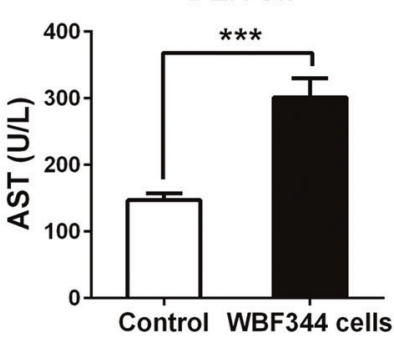

H
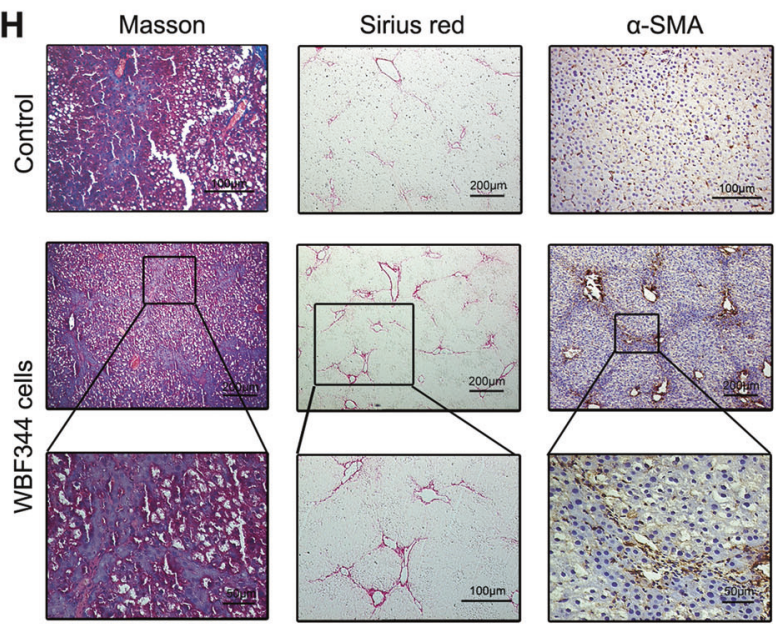

I
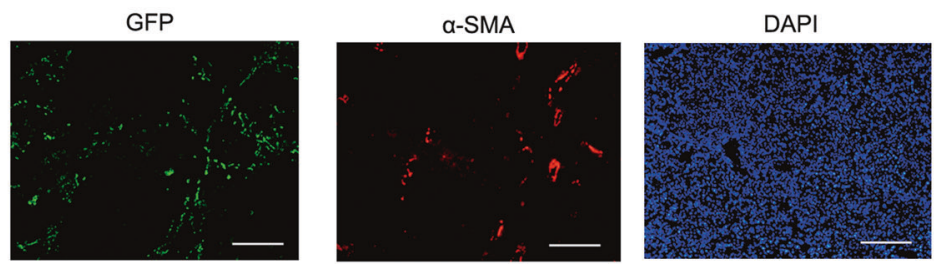
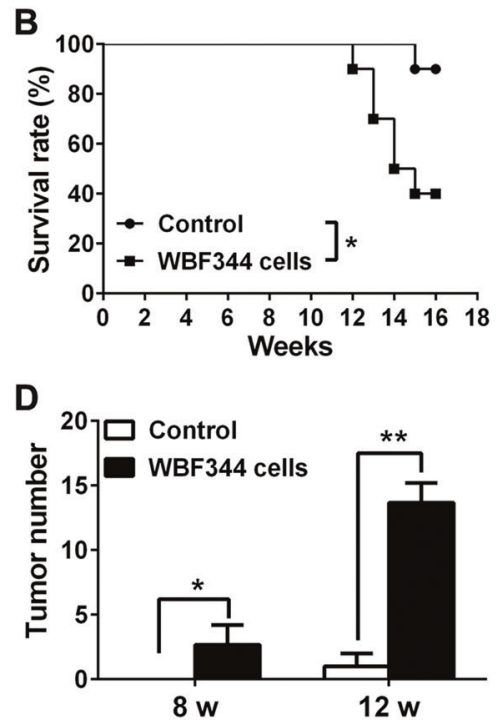

G

ow

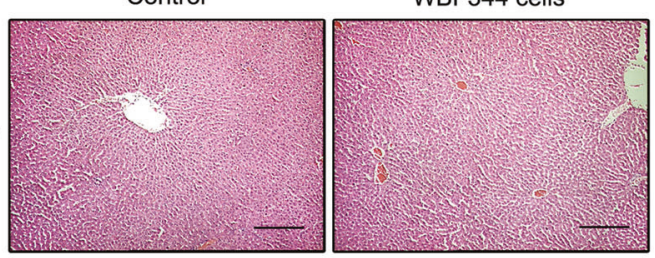

$8 \mathrm{~W}$
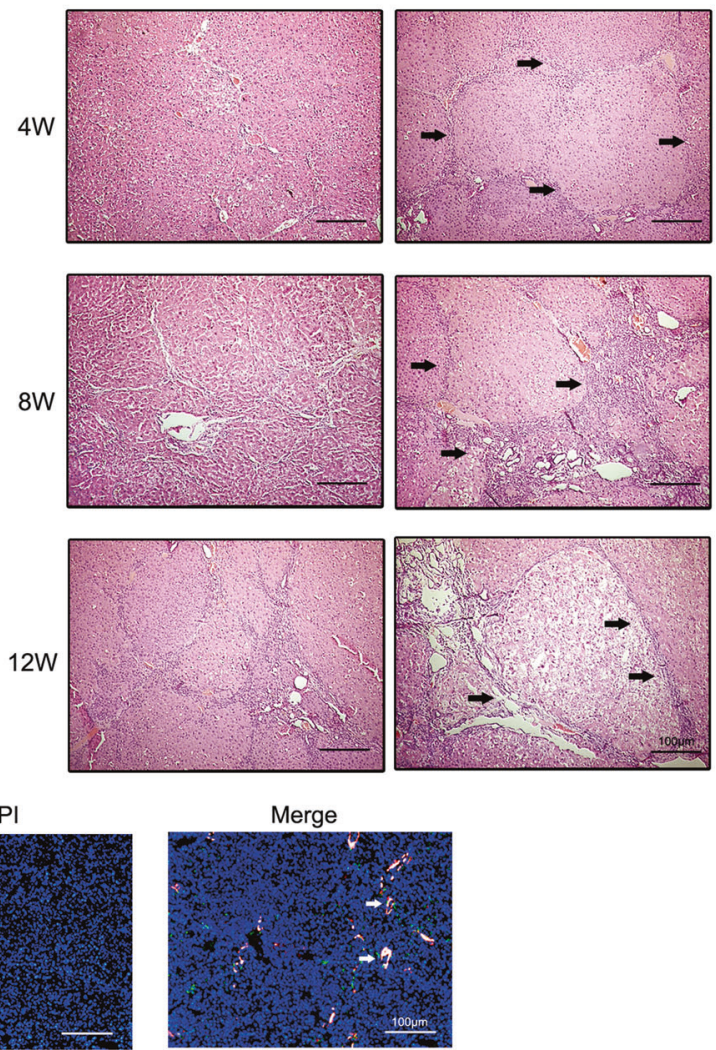

lead to upregulation of cancer-associated pathways in HPCs (Supplementary Fig. 4b, c). Interestingly, the upregulated genes were mostly associated with hepatic fibrosis (Fig. 5e, f). Additionally, expression of epithelialmarker was repressed, whereas mesenchymal cell markers exhibited high expression (Fig. 5g, h). As shown in Fig. 5i, the 
Fig. 2 Hepatic progenitor cell (HPC) transplantation promoted hepatocarcinogenesis and liver fibrosis in primary liver cancer model. a F344 rats were intrasplenically injected with HPC-like WB-F344 cells $\left(1 \times 10^{6} / 200 \mu \mathrm{l}\right)$ at 2 weeks after diethylnitrosamine $(\mathrm{DEN})$ treatment and sacrificed at different time points to observe the development of hepatocellular carcinoma (HCC). b DEN-treated F344 rats injected with WB-F344 cells; survival rate was observed. Data are represented as mean \pm SD. ${ }^{*} p<0.05$. c, $\mathbf{d}$ Rat livers of the indicated groups. The number of HCC nodules per liver in rats was determined at 8 and 12 weeks after DEN treatment. Data are represented as mean \pm SD. $* p<0.05, * * p<0.01$. e, f Serum levels of aspartate aminotransferase (AST) and alanine aminotransferase (ALT) were determined to indicate the extent of liver damage caused by DEN administration and the role of HPCs in promoting the damage. Data are represented as mean \pm SD. $* * p<0.01, * * * p<0.001$. g Hematoxylin and eosin $(\mathrm{H} \& \mathrm{E})$ staining was employed to observe the histological structure in different groups. h Hepatic collagen deposition was determined by Sirius Red staining and Masson's trichrome staining. The expression of $\alpha$-smooth muscle actin ( $\alpha$-SMA) was detected by immunohistochemical analysis. i WB-F344 cells were transfected with GFP-labeled lentiviral vector. After transplantation in rats, WB-F344 cells in the liver were detected by immunofluorescence double staining of GFP and $\alpha$-SMA expression. The merged immunofluorescence staining picture was used to determine the expression of $\alpha$-SMA in GFP-positive cells

expression of $\alpha$-SMA was significantly upregulated in LPStreated WB-F344 cells.

In order to confirm the mesenchymal differentiation of HPCs in vivo, the GFP-labeled HPCs were isolated from DEN-treated rat liver at 8 weeks by flow cytometry (Fig. 5j). As shown in Fig. 5k, $\alpha$-SMA expression was upregulated in GFP-positive cells compared with the control group. Furthermore, after homing in the microenvironment, the expression of epithelial cell markers in GFP-positive cells were repressed, whereas mesenchymal cells and myofibroblast markers exhibited high expression (Fig. 51-n). Taken together, these results suggest that LPS may induce the differentiation of HPCs into myofibroblasts.

\section{HPC-derived myofibroblasts produced high levels of IL- 6 and TNF- $a$, which promoted proliferation of HPCs via regulation of Ras and p53 signaling pathways by IncRNAs}

Having found that WB-F344 cell-derived myofibroblasts enhance the tumorigenic potential of HPCs, we collected conditioned medium (CM) from LPS-pretreated WB-F344 cells, which was then used to dispose the WB-F344 cells. Cell Counting Kit-8 (CCK8) assay showed that the CM of LPS-pretreated WB-F344 cells could significantly promote the proliferation of HPCs (Fig. 6a). Next, the Bio-Plex assay demonstrated that WB-F344 cell-derived myofibroblasts produced high levels of IL- 6 and TNF- $\alpha$ (Fig. 6b). To further investigate the mechanism of malignant transformation of HPCs, which was induced by WB-F344 cellderived myofibroblasts, microarray analysis of messenger
RNA (mRNA) expression was performed. The gene expression signature and KEGG (Kyoto Encyclopedia of Genes and Genomes) enrichment analysis showed that the Ras signaling was upregulated and p53 signaling was downregulated in WB-F344 cells treated with CM from WB-F344 cell-derived myofibroblasts (Fig. 6c, d). We next used reverse transcription-polymerase chain reaction (RTPCR) to validate the expression of Ras and p53 signaling pathway-related genes; epidermal growth factor receptor $(E G F R)$ was found to be upregulated and phosphatase and tensin homolog (PTEN) was found to be downregulated, most significantly (greater than six-fold) (Fig. 6e). Therefore, these two genes may play an important role in the malignant transformation of HPCs.

Next, lncRNA expression profile was analyzed in WBF344 cells, and then treated with CM of WB-F344 cellderived myofibroblasts. The relationship between the lncRNAs and mRNAs of EGFR and PTEN was determined. Compared to the control, ten IncRNAs that regulated both EGFR and PTEN expressions were found to be obviously upregulated (Fig. 6f). RT-PCR assay further confirmed that lncRNA-Ell2 and Dlg4 were the two most upregulated lncRNAs (Fig. 6g). Taken together, these results indicate that LPS induce HPC differentiation into myofibroblasts and produce high levels of IL- 6 and TNF- $\alpha$, which lead to the aberrant expression of Ras and p53 cancer-related pathways, by IncRNA regulation.

\section{LPS/TLR4 signaling pathway contributed to IL- 6 and TNF-a production in HPC-derived myofibroblasts, and malignant transformation of HPCs}

TLR4 is an important receptor of LPS; immunofluorescence staining showed that TLR4 was expressed at a high level in WB-F344 cells (Supplementary Fig. 5a). We silenced TLR4 expression in WB-F344 cells by using three short hairpin RNA (shRNAs). As shown in Supplementary Fig. 5b, c, TLR4 shRNA3 demonstrated the best suppression efficiency. Further data demonstrated that TLR4 silencing diminished the secretion of IL- 6 and TNF- $\alpha$ in LPS-disposed WB-F344 cells (Supplementary Fig. 5d). Thereafter, compared to the control group, the CM collected from the LPS-disposed TLR4 silencing WB-F344 cells could not enhance the proliferation of WB-F344 cells (Supplementary Fig. 5e). Additionally, TLR4 silencing in LPS-disposed WB-F344 cells diminished the ability of tumor formation of HPCs in vivo (Supplementary Fig. 5f, g). Consistent with this finding, liver fibrosis and hepatocarcinogenesis were also inhibited by TLR4 deletion (Supplementary Fig. 5h, i). These results indicate that LPS/TLR4 plays an important role in the differentiation from HPCs into myofibroblasts and increases the production of IL- 6 and TNF- $\alpha$, which finally 
A

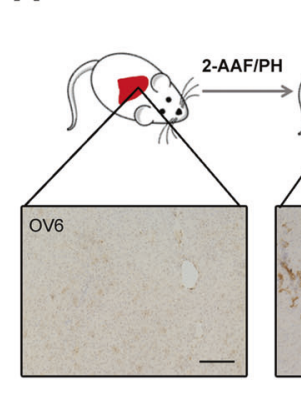

C

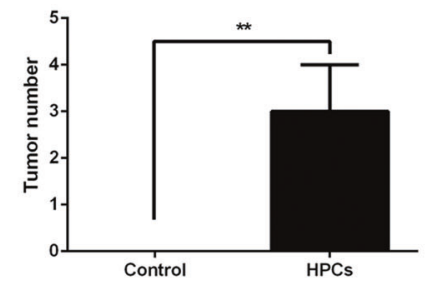

$\mathbf{F}$

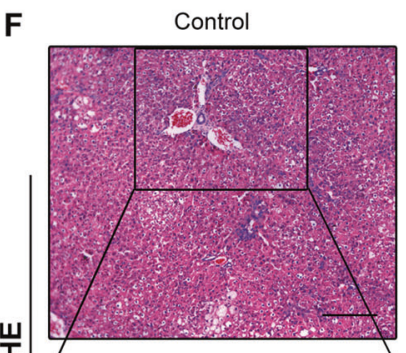

필

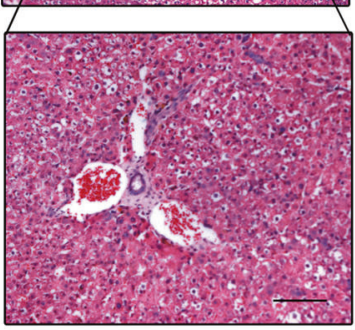

H
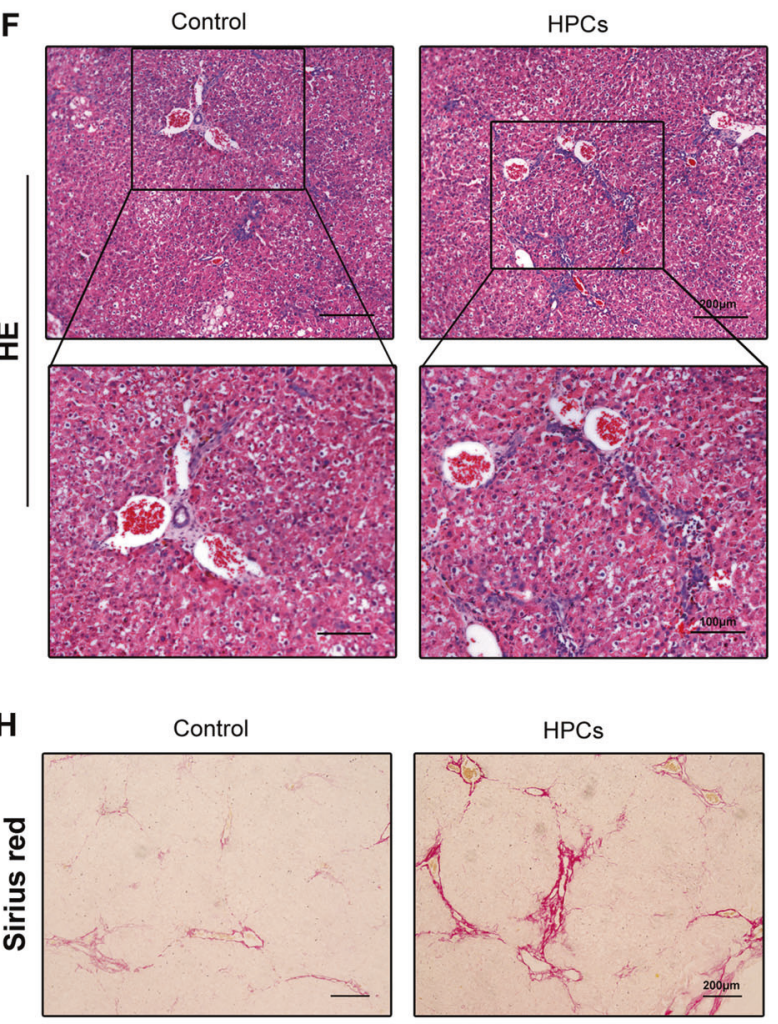

Fig. 3 Primary hepatic progenitor cell (HPC) transplantation promoted hepatocarcinogenesis and liver fibrosis in primary hepatocellular carcinoma (HCC) model. a Primary HPCs were isolated from 2-acetylaminofluorine/partial hepatectomy (2-AAF/PH) model. b, c Rat livers of the indicated groups. The number of HCC nodules per liver in rats was determined at 8 weeks after diethylnitrosamine (DEN) treatment. Data are represented as mean $\pm \mathrm{SD}$. ${ }^{* *} p<0.01$. d, e Serum levels of aspartate aminotransferase (AST) and alanine
B

DEN 8w
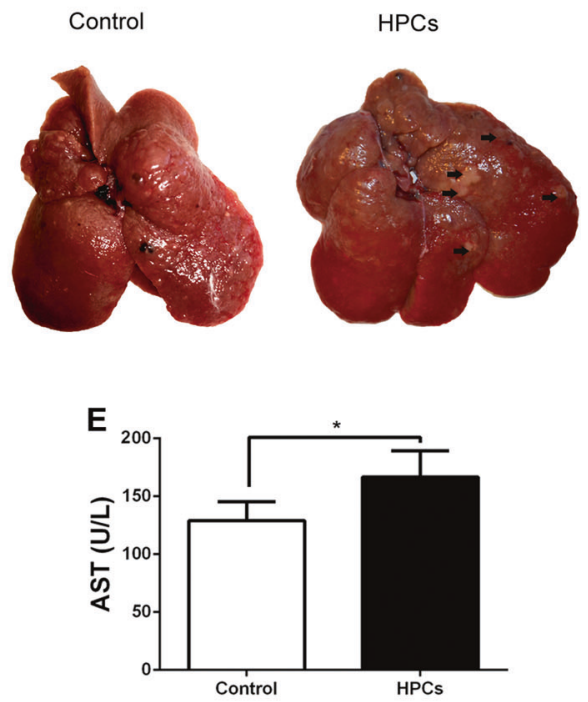

G
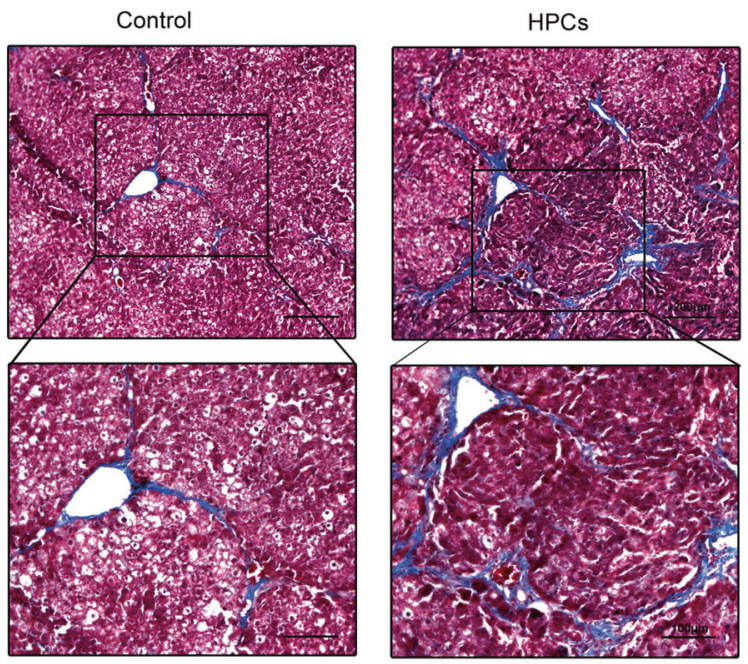

I
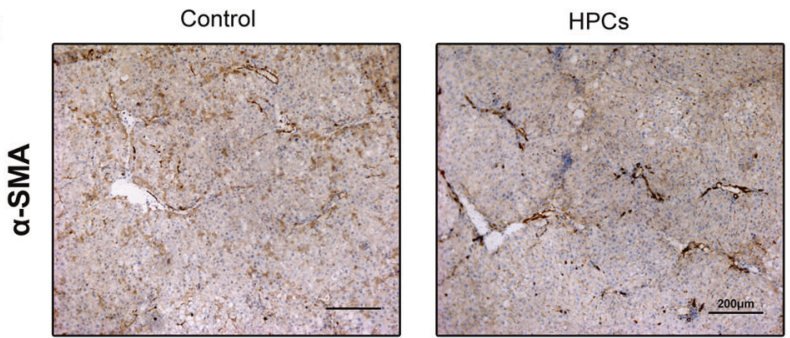

aminotransferase (ALT) were determined to indicate the extent of liver damage caused by DEN administration and the role of HPCs in promoting the damage. Data are represented as mean \pm SD. ${ }^{*} p<0.05$. f Hematoxylin and eosin (H\&E) staining was employed to observe the histological structure in different groups. g-i Hepatic collagen deposition was determined by Sirius Red staining and Masson's trichrome staining. The expression of $\alpha$-smooth muscle actin ( $\alpha$-SMA) was detected by immunohistochemical analysis 

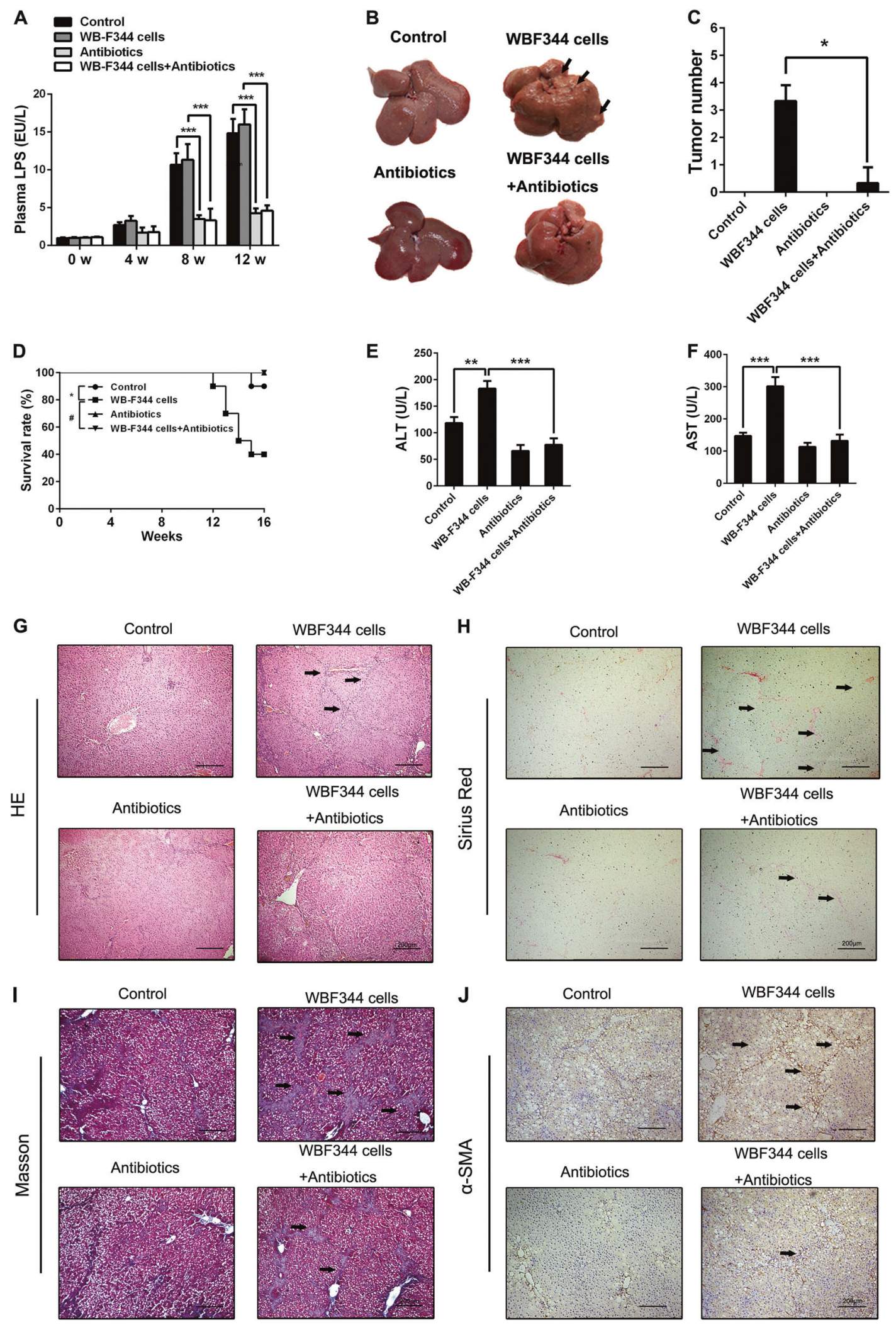
Fig. 4 Lipopolysaccharide (LPS) elimination alleviated hepatic progenitor cell (HPC) transplantation-induced hepatocarcinogenesis and liver fibrosis in primary hepatocellular carcinoma (HCC) model. a A combination of four different antibiotics, including ampicillin $(1 \mathrm{~g} / \mathrm{l}$; Sigma), neomycin sulfate ( $1 \mathrm{~g} / \mathrm{l}$; Sigma), metronidazole (1 g/l; Sigma), and vancomycin $(500 \mathrm{mg} / \mathrm{l}$; Abbott Labs) was used to eliminate gutderived LPS during HCC occurence. Serum LPS levels were measured by enzyme-linked immunosorbent assay (ELISA) in the indicated groups. Data are represented as mean $\pm \mathrm{SD}$. $* * * p<0.001$. b, c Rat livers of the indicated groups. The number of HCC nodules per liver in rats was determined at the eighth week after diethylnitrosamine (DEN) treatment. Data are represented as mean \pm SD. $* p<0.05$. d Survival rate was observed. LPS elimination alleviated the death in DENtreated rats. Data are represented as mean \pm SD. $* p<0.05$ and ${ }^{\#} p<$ 0.01. e, f Serum levels of AST and ALT were determined to indicate the extent of liver damage. Data are represented as mean \pm SD. ${ }^{* *} p<$ 0.01 and $* * * p<0.001$. g Hematoxylin and eosin (H\&E) staining was employed to observe the histological structure in different groups. $\mathbf{h}-\mathbf{j}$ Hepatic collagen deposition was determined by Sirius Red staining and Masson's trichrome staining. Expression of $\alpha$-smooth muscle actin $(\alpha-$ SMA) was detected by immunohistochemical analysis

leads to the promotion of liver fibrosis and hepatocarcinogenesis in DEN-induced HCC model.

\section{IL-6 and TNF-a level in peritumoral tissue correlated with HPCs activation, hepatic fibrosis, and HCC recurrence}

We next explored the correlation of IL- 6 and TNF- $\alpha$ level with HPC activation, hepatic fibrosis, and HCC incidence in HCC peritumoral specimens. IL- $6^{\text {low }}$ TNF- $\alpha^{\text {low }}$, IL- $6^{\text {low }}$ TNF$\alpha^{\text {high }}$, IL- $6^{\text {high }}$ TNF- $\alpha^{\text {low }}$, and IL- $6^{\text {high }}$ TNF- $\alpha^{\text {high }}$ were observed in 26 of 84 (30.9\%), 15 of 84 (17.8\%), 18 of 84 (21.4\%), and 25 of $84(29.8 \%)$ cases, respectively (Fig. 7a, b). We further determined the OV6 and $\alpha$-SMA expression in the four groups and found that IL- $6^{\text {high }}$ TNF- $\alpha^{\text {high }}$ group had the highest level of OV6 and $\alpha$-SMA expression, compared to the other three groups (Fig. 7c, d). The results indicate that IL- 6 and TNF- $\alpha$ production is associated with quantity of activated HPCs and myofibroblasts. Besides, recurrence rate in IL- $6^{\text {high }}$ TNF- $\alpha^{\text {high }}$ group was significantly higher than the other groups (Fig. 7e), which indicates that the expression of IL- 6 and TNF- $\alpha$ in peritumoral tissues may be useful in predicting recurrence of $\mathrm{HCC}$.

In summary, we found that HPCs could promote hepatic fibrosis and hepatocarcinogenesis. Furthermore, LPS induced differentiation of HPCs into myofibroblasts, resulting in the proliferation and malignant transformation of HPCs. The potential mechanism underlying the process of hepatocarcinogenesis by HPCs is that LPS/ TLR4 signaling induced the differentiation of HPCs into myofibroblasts, with subsequent production of IL- 6 and TNF- $\alpha$. These cytokines, in turn, promoted the upregulation of EGFR and downregulation of PTEN, through lncRNA regulation, resulting in the aberrant expression of Ras and p53 signaling pathways, which finally enhanced proliferation and malignant transformation of HPCs (Fig. 7f).

\section{Discussion}

It has been reported that the degree of HPC activation positively correlates with the severity of liver disease $[22,23]$. In chronic hepatitis, activation of HPCs correlates with the degree of inflammation and fibrosis, and stage of disease [23-25]. In the current study, we also found that HPC activation correlated with hepatic fibrosis and hepatocarcinogenesis. In vivo experiments indicated that HPC transplantation promoted liver fibrosis and HCC in the DEN model, suggesting that HPCs are instrumental in aggravating the liver damage in chronic liver injury processes. We traced the fate of HPCs in vivo by transfecting GFP lentiviral vector in WB-F344 cells and the results showed that GFP-positive cells formed the liver fibrosis lesions, indicating that HPCs may differentiate into myofibroblasts.

In majority of patients, primary liver cancer arises in an inflammation-associated microenvironment. LPS is commonly associated with a variety of human liver disorders, including liver fibrosis and HCC that leads to severe inflammation by activating various inflammatory cells [26, 27]. In the present study, the level of LPS continued to elevate in rats with DEN treatment. At this point, it is unclear whether LPS plays a role in the promotion of liver damage by HPC transplantation. Our group has earlier reported that LPS could regulate the metastatic and stemness potential of HCC cells and liver cancer stem cells $[28,29]$. However, the effect of LPS on HPCs has not yet been fully understood. In the current study, we found that the decrease of LPS level diminished the effect of HPC transplantation on liver fibrosis and hepatocarcinogenesis. Notably, LPS preconditioning of HPCs did not augment its tumorigenic potential in vivo. However, these cells could promote the tumorigenic potential of normal HPCs, which meant that LPS-pretreated HPCs differentiated into myofibroblasts and then enhanced the tumorigenicity of HPCs, resulting in the promotion of HCC. Taken together, these findings indicate that LPS has a significant role in liver fibrosis and hepatocarcinogenesis promoted by HPCs.

At this point, it is unclear whether genetic alterations have occurred in HPCs preconditioned with LPS. Transcriptomic analysis revealed that LPS-induced HPCs are related to myofibroblasts, indicated by the expression of several markers, such as CTGF, COL1A1, COL4A1, and $T G F-\beta$. Additionally, the change of epithelia and mesenchymal cell markers was observed in LPSpreconditioned HPCs, which gave HPCs the mesenchymal cell phenotype. It is well known that HCC development is mediated by the contact between stromal and tumor cells. In 
A
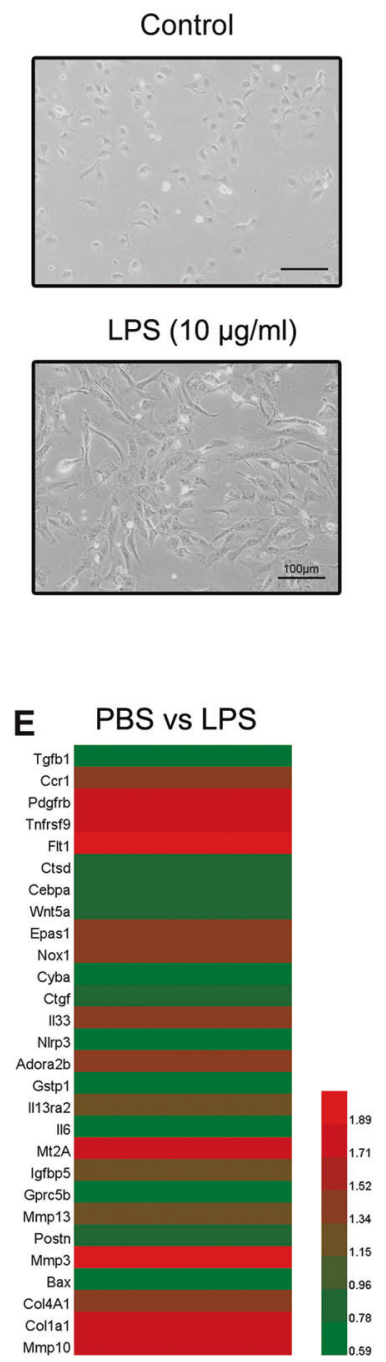

K

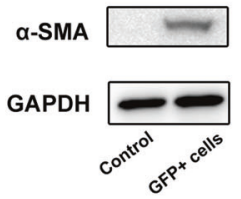

B

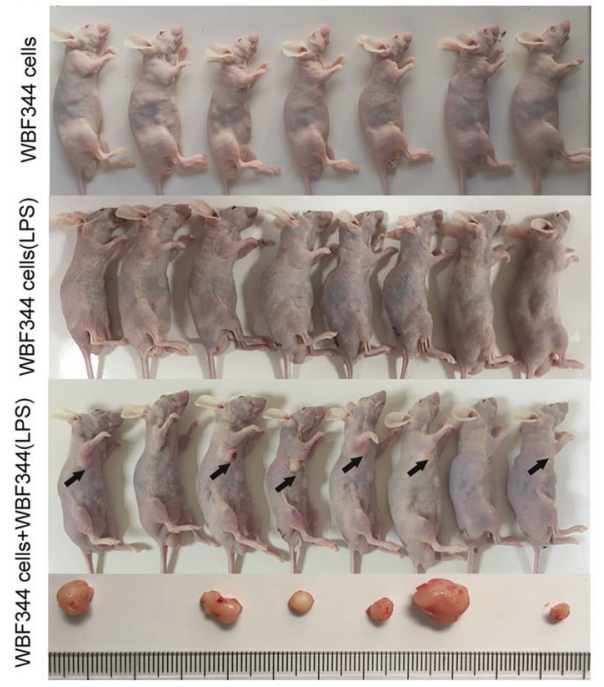

C

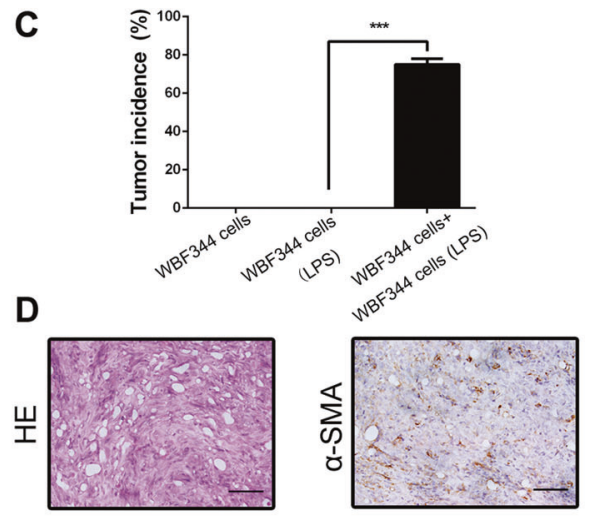

D

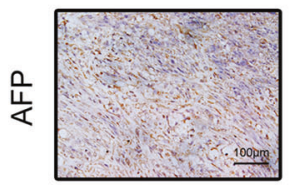

F

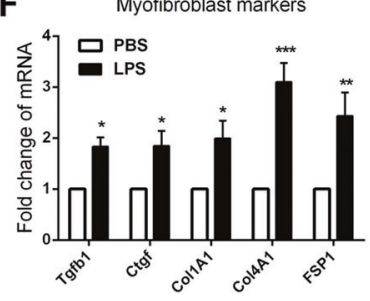

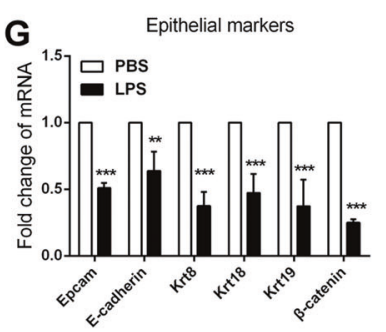

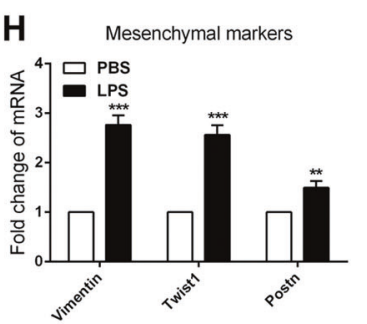

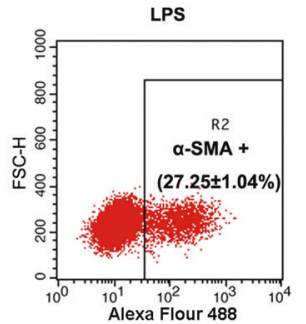

J

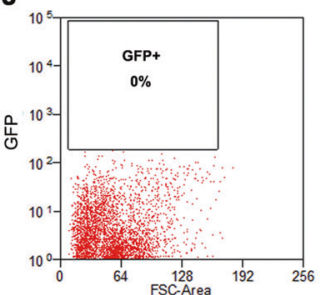

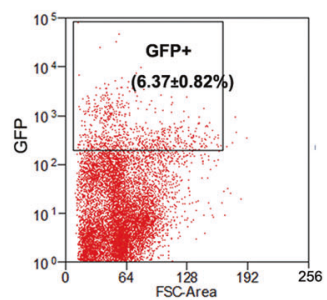

the present study, we found that HPC-derived myofibroblasts could significantly promote the proliferation of HPCs. During the transformation of HPCs to cancer cells, stromal cells expressed pro-inflammatory mediators, such as IL-6 and TNF- $\alpha$, which were found to play an important role in HPC proliferation and abnormal differentiation [30-32]. In our study, we found that LPS induced HPC differentiation into myofibroblasts, followed by subsequent secretion of high levels of IL- 6 and TNF- $\alpha$, which might be involved in the proliferation and malignant transformation of HPCs.
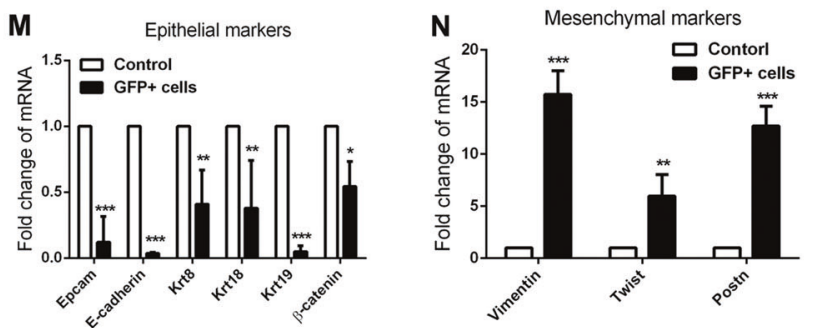

Recent studies have confirmed the upregulation of IL-6 and TNF- $\alpha$ in human HCC, and suggested that they played a central role in the gene expression network that drived tumor development [31, 33]. IL-6 and TNF- $\alpha$ have also been reported to promote HPC activation and proliferation during HCC formation [34]. To further clarify the mechanism of HPC proliferation and malignant differentiation induced by IL- 6 and TNF- $\alpha$, the mRNA expression profile was analyzed in HPCs treated with conditioned medium of HPC-derived myofibroblasts. We found that the 
Fig. 5 Lipopolysaccharide (LPS) induced hepatic progenitor cell (HPC) differentiation into myofibroblasts, which enhanced the tumorigenic potential of HPCs. a Cell morphology was observed in LPS-disposed WB-F344 cells for 2 weeks. b, c Tumorigenicity was detected in each group. Pictures were taken at the eighth week after subcutaneous injection. d Subcutaneous tumor was observed with hematoxylin and eosin (H\&E) staining. $\alpha$-Fetoprotein (AFP) and $\alpha-$ smooth muscle actin ( $\alpha$-SMA) expression was detected by immunohistochemical analysis. e RNA was extracted from WB-F344 cells after 2 weeks of LPS exposure and subjected to microarray analysis ( $n$ $=3$ for each sample). Heatmap displaying positive fold changes in expression of myofibroblast-like genes in phosphate-buffered saline (PBS)-treated WB-F344 cells versus LPS-treated WB-F344 cells. f Expression of myofibroblast-associated genes was examined by realtime PCR $(n=3$; mean $\pm \mathrm{SD}) . * p<0.05, * * p<0.01$, and $* * * p<$ 0.001. g, h Expression of epithelial and mesenchymal markers were examined by real-time PCR $(n=3$; mean $\pm \mathrm{SD}) . * * p<0.01$ and $* * * p$ $<0.001$. i Flow cytometry was performed to analyze $\alpha$-SMA expression in PBS-treated WB-F344 cells versus LPS-treated WB-F344 cells $(n=3$; mean $\pm \mathrm{SD})$. j WB-F344 cells were transfected with GFPlabeled lentiviral vector. Then, F344 rats were intrasplenically injected with GFP-labeled WB-F344 cells at 2 weeks after DEN treatment. After transplantation in DEN-induced rats for 6 weeks, GFP-positive cells were isolated from the liver by using flow cytometry. $\mathbf{k}$. Western blot was employed to detect $\alpha$-SMA expression in GFP-positive cells and WB-F344 cells. 1 Expression of myofibroblast-associated genes in GFP-positive cells and WB-F344 cells was examined by real-time PCR $(n=3$; mean $\pm \mathrm{SD}) . * * p<0.01$ and $* * * p<0.001$. $\mathbf{m}$, n Expression of epithelial and mesenchymal markers in GFP-positive cells and WB-F344 cells were examined by real-time PCR $(n=3$; mean \pm SD). $* p<0.05, * * p<0.01$, and $* * * p<0.001$

classical cancer-related signaling pathways, Ras and p53, were aberrantly expressed in HPCs treated with LPSdisposed HPCs, indicating that IL- 6 and TNF- $\alpha$ promoted the proliferation and malignant transformation of HPCs through deregulation of Ras and p53 signaling pathways. $E G F R$ was found to be most significantly upregulated, whereas PTEN was found to be the most significantly downregulated. The aberrant expression of EGFR and $P T E N$ has been reported to lead to cell proliferation, differentiation, and survival in cancer [35, 36]. Our studies indicate that the critical role of EGFR and PTEN expression shifts from HPCs to cancer cells during the course of DENinduced liver tumorigenesis. Indeed, during HCC formation, HPCs might be the origin of both tumor-associated stromal cells and tumor cells.

Emerging evidence has indicated a regulatory role of lncRNAs in various cancers, including $\operatorname{HCC}[37,38]$. However, knowledge on the role of IncRNAs in the expansion and malignant transformation of HPCs remains largely unclear. Herein, we analyzed lncRNA expression profile in HPCs treated with conditioned medium of HPCderived myofibroblasts. We found that ten IncRNAs, which targeted both EGFR and PTEN, were upregulated, amongst which expression of lncRNA-Ell2 and $\operatorname{lncRNA-Dlg4}$ was most significantly increased. Therefore, our data suggest that IL-6 and TNF- $\alpha$ from HPC-derived myofibroblasts upregulated lncRNA-Ell2 and Dlg4, which resulted in the aberrant expression of EGFR and PTEN and the deregulation of Ras and P53 signaling pathways, and finally led to the promotion of HPC proliferation and malignant transformation.

Activation of LPS/TLR4 signaling leads to the promotion of cell survival and proliferation in $\operatorname{HCC}[16,39]$. However, the role of LPS/TLR4 signaling pathway in HPC differentiation is unclear. We now found that the expression of TLR4 was high in HPCs; LPS-induced IL- 6 and TNF- $\alpha$ production in HPCs was decreased, when TLR4 expression was silenced. Moreover, HPC proliferation and malignant transformation were also diminished, when treated with the CM of LPS-disposed HPCs without TLR4 expression. These results suggest TLR4 plays a key role in mediating LPS-induced HPC differentiation into myofibroblasts and the production of IL- 6 and TNF- $\alpha$, which finally leads to the promotion of liver fibrosis and hepatocarcinogenesis.

In summary, we identify an indispensable role of LPS/ TLR4 in the differentiation of HPCs to myofibroblasts, and the increased secretion of IL- 6 and TNF- $\alpha$. Moreover, our results suggest that IL- 6 and TNF- $\alpha$ are the essential factors of neoplastic transformation in HPCs, which leads to the aberrant expression of Ras and p53 signaling pathways. This finding will further promote our understanding about regulation of HPC differentiation and tumorigenesis in the liver.

\section{Materials and methods}

\section{Patients and tissue specimens}

Specimens of HCC tissues were obtained from 84 HCC patients who underwent hepatic resection at the Shanghai Eastern Hepatobiliary Surgery Hospital from 1997 to 2007. These patients included 75 men and 9 women, with a median age of 41.59 years (range: 21-71), and all the specimens were subjected to IHC analysis. Prior informed consent was obtained, and the study protocol was approved by the Ethics Committee of the Shanghai Eastern Hepatobiliary Surgery Hospital.

\section{Cell culture and treatment}

WB-F344 cells were incubated in Dulbecco's modified Eagle's medium (DMEM, Gibco-BRL, Gaithersburg, MD, USA), containing $10 \%$ FBS and $100 \mathrm{mg} / \mathrm{l}$ penicillin, and $100 \mathrm{mg} / \mathrm{l}$ streptomycin. WB-F344 cells were treated with LPS (Sigma, Saint Louis, MO, USA) at $10 \mu \mathrm{g} / \mathrm{ml}$ concentration, for 2 weeks. 
A

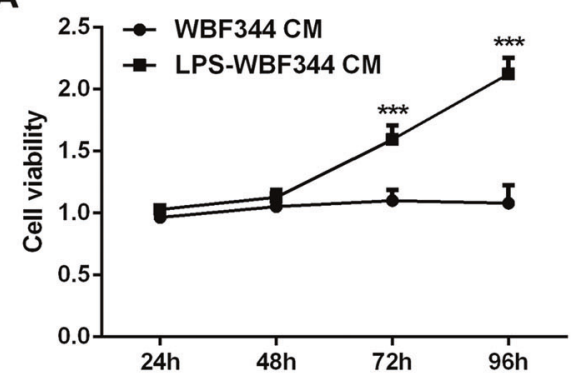

C

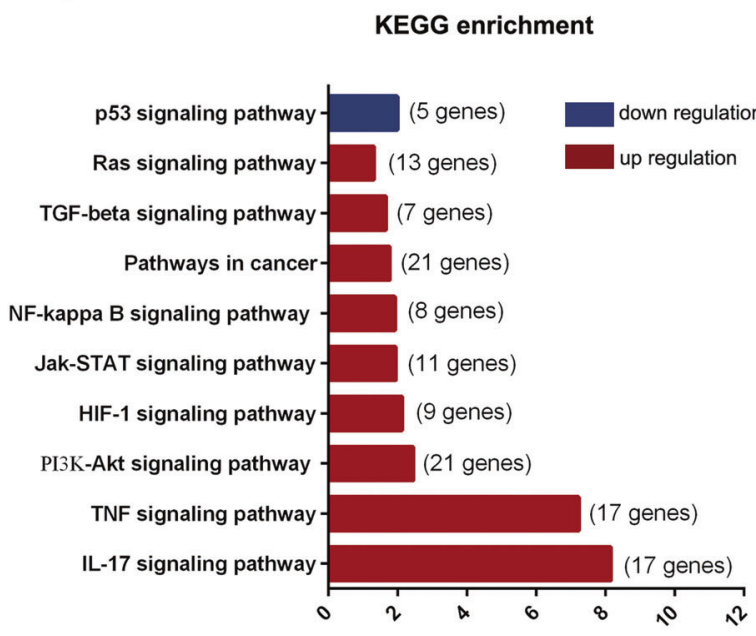

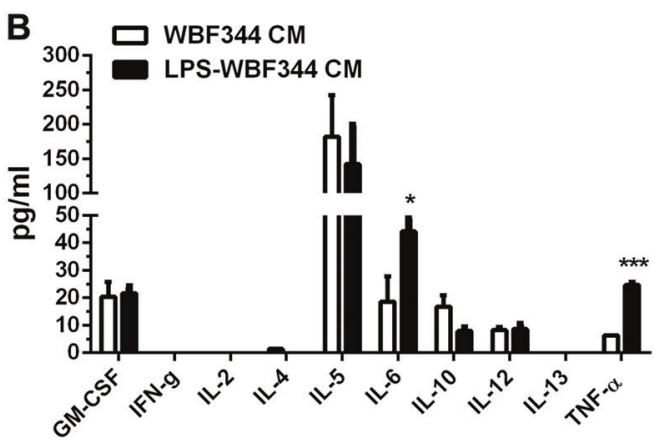

D WBF 344 CM vs LPS-WBF $344 \mathrm{CM}$

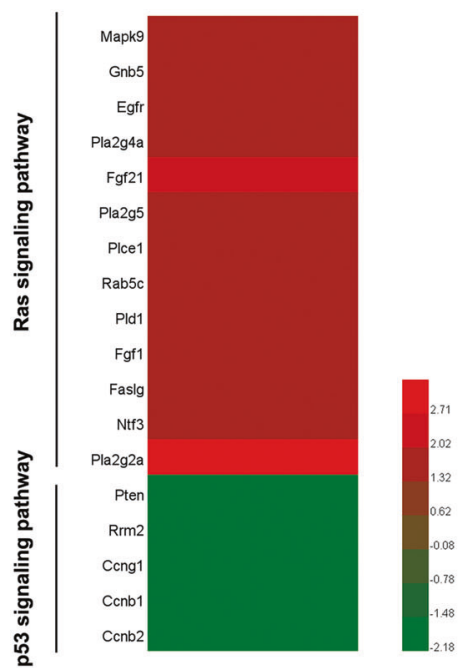

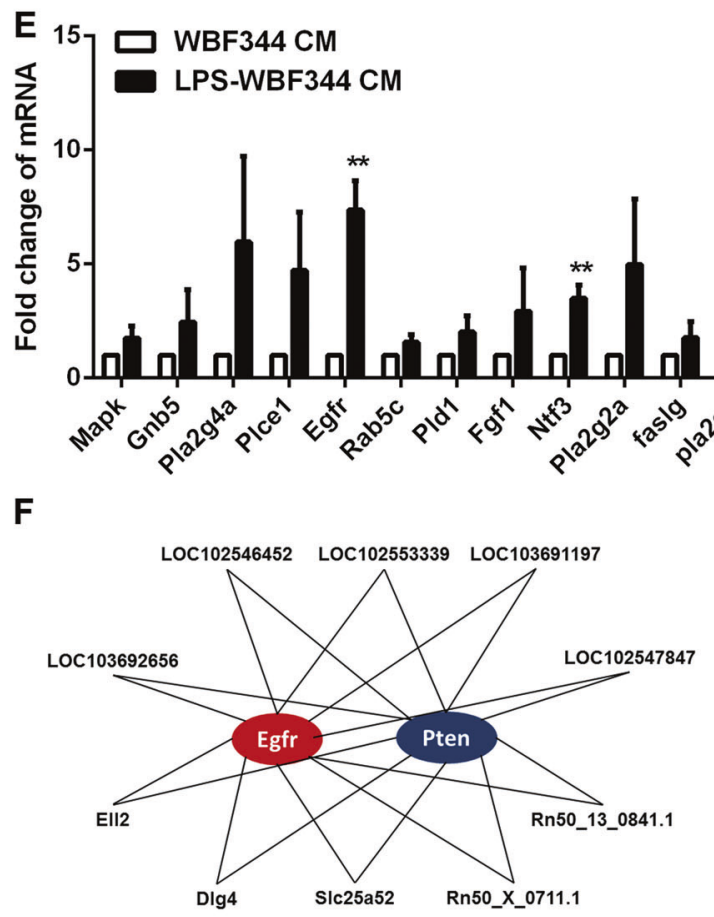

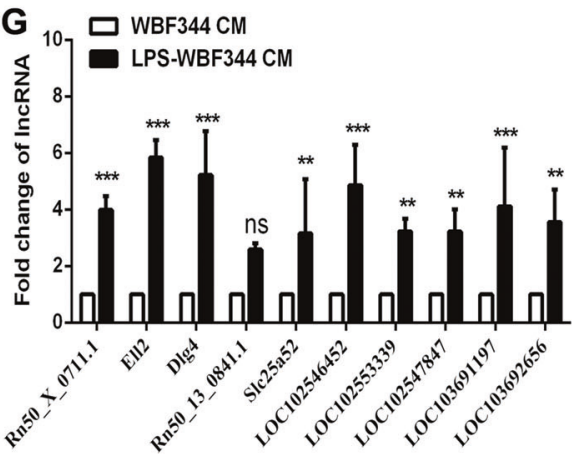


Fig. 6 Hepatic progenitor cell (HPC)-derived myofibroblasts produced high levels of interleukin-6 (IL-6) and tumor necrosis factor- $\alpha$ (TNF$\alpha$ ), which promoted proliferation of HPCs by regulating epidermal growth factor receptor (EGFREGFR) and phosphatase and tensin homolog (PTEN) expression via long non-coding RNAs (lncRNAs). a Cell proliferation was detected by Cell Counting Kit-8 (CCK8) assay in WB-F344 cells treated with lipopolysaccharide (LPS)-pretreated WB-F344 cells conditioned medium (CM) and control $(n=3$; mean \pm SD). $* * * p<0.001$. b Inflammatory factors were examined by Bio-Plex assay $(n=3$; mean $\pm \mathrm{SD})$. $* p<0.0$, and $* * * p<0.001$. c RNA was extracted from WB-F344 cells after treatment of LPS-pretreated WBF344 cells CM and subjected to microarray analysis $(n=3$ for each sample). The top 10 pathways enriched among the upregulated messenger RNAs (mRNAs) (red) and downregulated mRNAs (blue) in WB-F344 cells. d Heatmap displaying fold changes in expression of Ras and p53 signaling pathways in WB-F344 cells after treatment of LPS-pretreated WB-F344 cells CM and phosphate-buffered saline (PBS)-pretreated WB-F344 cells CM. e Expression of selected genes of Ras and p53 signaling pathway was examined by real-time PCR ( $n$ $=3$; mean $\pm \mathrm{SD}$ ). $* p<0.05, * * p<0.01$, and $* * * p<0.001$. f The network represents co-expression correlations between the two mRNAs and upregulated lncRNAs. $g$ Expression of selected genes of Ras and p53 signaling pathways was examined by real-time PCR $(n=3$; mean $\pm \mathrm{SD}) . * * p<0.01$ and $* * * p<0.001 ;$ ns, not significant

\section{Animal models}

Male F344 rats (8-10 weeks old, weighing 160-180 g) were obtained from Shanghai Laboratory Animal Center (Shanghai, China), and were housed in a pathogen-free animal facility. Rats received DEN at $0.01 \%$ concentration, through drinking water. After 2 weeks, single-cell suspensions $\left(10^{6}\right.$ WB-F344 cells/200 $\mu \mathrm{l}$ saline solution) were i.s. injected into the DEN-pretreated rats. Blood samples were collected and serum was separated for biochemical analysis at the indicated time. The serum levels of ALT and AST were determined. Meanwhile, rats were sacrificed to obtain liver samples. The animal protocols were approved by Second Military Medical University Animal Care Committee.

\section{Histological examination}

Liver samples were fixed in $4 \%$ paraformaldehyde, paraffin embedded, and sectioned. H\&E, Sirius Red, and Masson's trichrome staining were performed. Each sample was independently assessed and scored by three pathologists, blinded to the study protocol.

\section{IHC staining and immunofluorescence}

IHC analysis was performed using the following antibodies: rabbit anti- $\alpha$-SMA (diluted 1:100, Abcam, Cambridge, UK), anti-AFP (diluted 1:200, Abcam, Cambridge, UK), anti-OV6 (diluted 1:100, Abcam, Cambridge, UK), anti-IL6 (diluted 1:200, Abcam, Cambridge, UK), and anti-TNF- $\alpha$ (diluted 1:200, Abcam, Cambridge, UK). The detailed method has been published previously [12]. Five fields in each section were randomly selected to calculate the ratio of positive expression area. The density of immunostaining was measured using Image-Pro Plus, Version 6.2 software (Media Cybernetics Inc., Bethesda, MD, USA), described previously as an established method [40]. The IOD in each image was measured, and the density of each marker was calculated as IOD/total area of each image. For immunofluorescence, mouse anti-GFP (diluted 1:200, CST, USA), rabbit anti- $\alpha$-SMA (diluted 1:100, Abcam, Cambridge, UK), and rabbit anti-TLR4 (diluted 1:200, Abcam, Cambridge, UK) were used.

\section{Isolation of primary HPCs}

To isolate primary HPCs, 2-AAF/PH was employed in Sprague-Dawley (SD) rats. 2-AAF was administered daily at a dose of $10 \mathrm{mg} / \mathrm{kg}$ for 4 days. On the fifth day, $70 \%$ hepatectomy was performed. 2-AAF was continued to be administered for 1 week. Under sterile conditions, SD rats were anesthetized. The pre-warmed GBSS (Gey's balanced salt solution) solution (containing $0.125 \mathrm{mg} / \mathrm{ml}$ DnaseI) was perfused into the liver via the portal vein, then $0.1 \%$ of type IV collagenase was perfused, and the liver was cut and placed in a $0.06 \%$ type IV collagenase solution. After shaking at $37^{\circ} \mathrm{C}$ for $30 \mathrm{~min}, 100$ mesh filter was filtered, placed in $0.01 \%$ protease solution, shaken at $37^{\circ} \mathrm{C}$ for $30 \mathrm{~min}$, centrifuged at $50 \times \mathrm{g}$ for $5 \mathrm{~min}$, the supernatant was collected, centrifuged at $350 \times g$ for $5 \mathrm{~min}$, and then resuspended in DMEM medium. OV6-positive cells were isolated from hepatic cell suspensions with immunomagnetic beads.

\section{In vivo tumorigenicity experiments}

Six-week-old male athymic BALB/c nu/nu mice were obtained from Shanghai Experimental Animal Center, Chinese Academy of Science. Mice were maintained under a pathogen-free condition and treated in accordance with the institutional animal welfare guidelines of the Second Military Medical University. For the assay to assess tumorigenicity, $5 \times 10^{6}$ WB-F344 cells, cultured with phosphatebuffered saline (PBS) or LPS $(10 \mu \mathrm{g} / \mathrm{ml})$ for 2 weeks, were injected subcutaneously into the left back of the mice. At the end of 8 weeks, the mice were sacrificed for analysis.

\section{Transcriptome sequencing}

Total RNA was extracted from each tissue sample using TRIzol (Life Technologies, Grand Island, NY, USA), according to the protocol provided by the manufacturer. Five micrograms of RNA of each sample was individually used for the construction of transcriptome libraries, using 
A

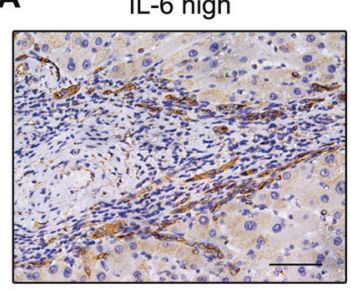

IL-6 low

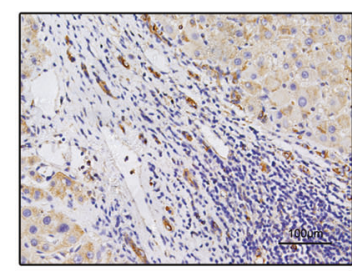

B TNF- $\alpha$ high

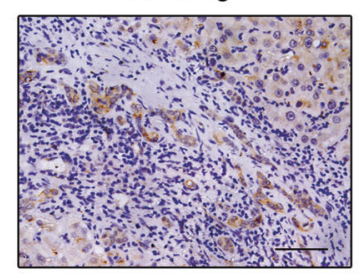

TNF- $\alpha$ low

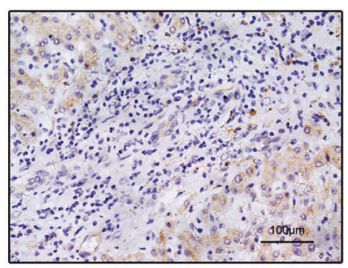

C

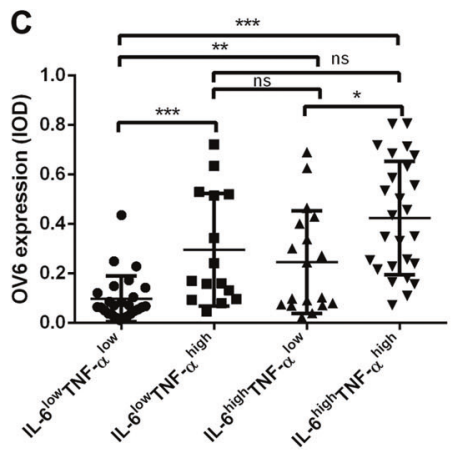

D

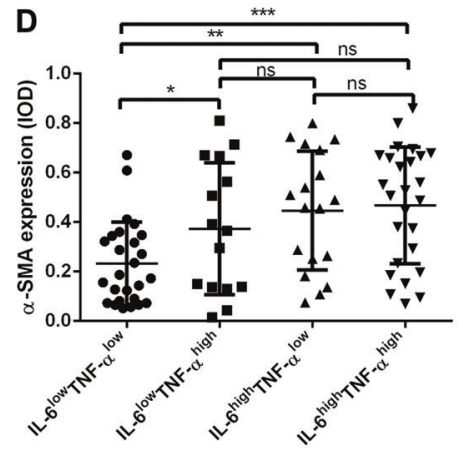

E

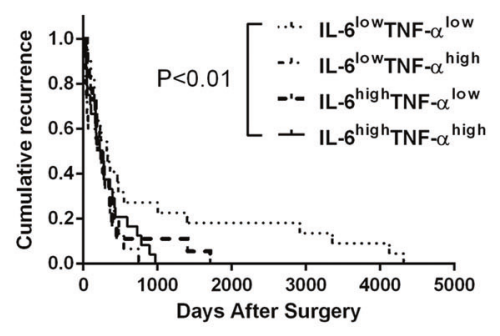

$\mathbf{F}$

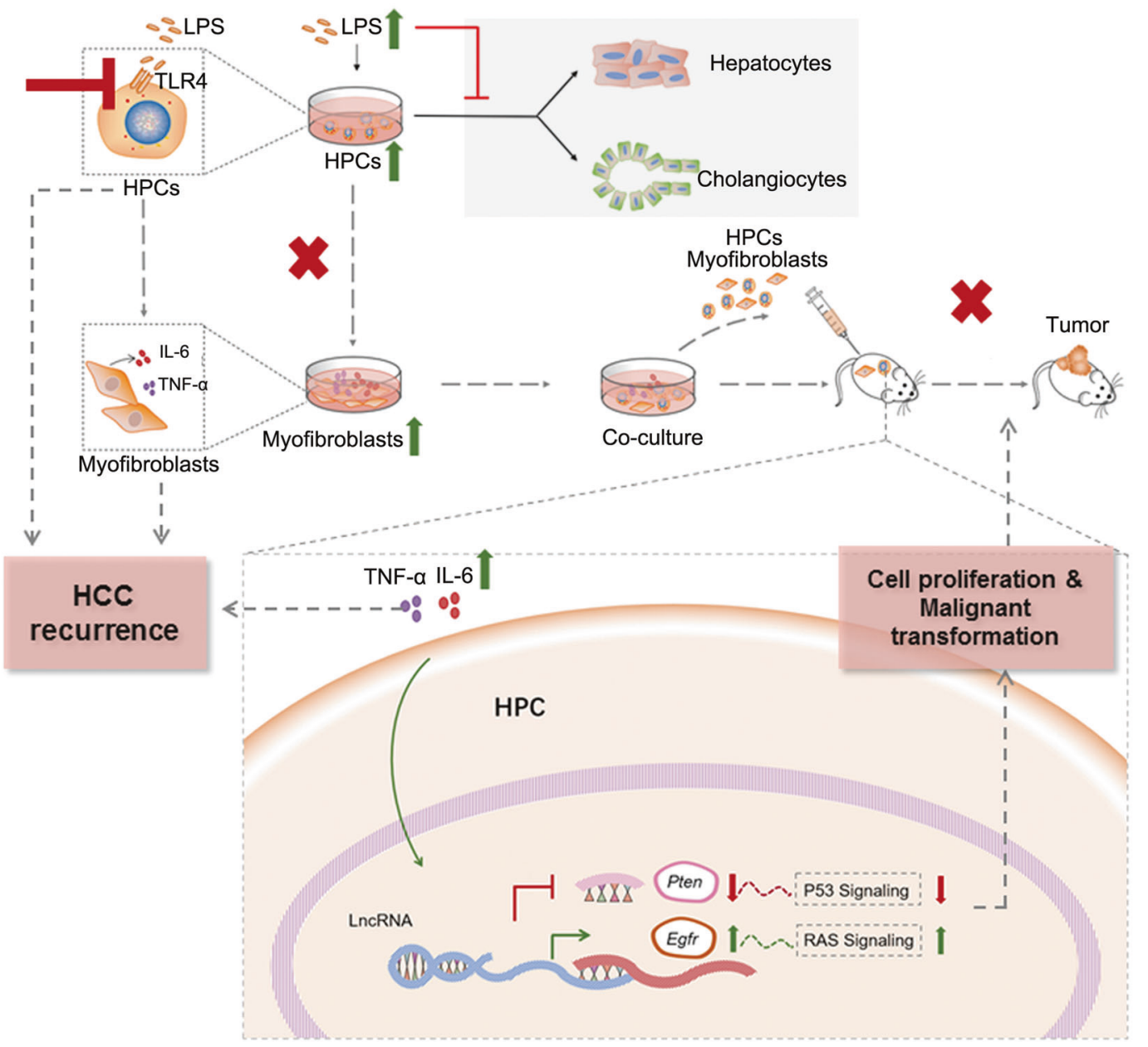

IlluminaTruSeq ${ }^{\mathrm{TM}}$ RNA Sample Preparation Kit (Illumina, San Diego, CA, USA), and sequenced using IlluminaHiSeq ${ }^{\mathrm{TM}} 2000$, according to the manufacturer's instructions. Q20 was used as quality control standard to filter raw reads. After filtering the low-quality reads, the adaptors of high-quality reads were removed, and then 
Fig. 7 Interleukin-6 (IL-6) and tumor necrosis factor- $\alpha($ TNF- $\alpha)$ level in peritumoral tissue correlated with hepatic progenitor cell (HPC) activation, hepatic fibrosis, and hepatocellular carcinoma (HCC) recurrence. a, b IL- 6 and TNF- $\alpha$ expression was detected in HCC peritumoral tissues by immunohistochemical analysis. c, d. OV6 and $\alpha$-smooth muscle actin ( $\alpha$-SMA) expression was analyzed in each group. $* p<0.05, * * p<0.01$, and $* * * p<0.001$. e. Recurrence rate was analyzed in each group of HCC patients (Kaplan-Meier method); $p$ values were obtained by log-rank multiple comparison tests. f A schematic model of the mechanism underlying the role of lipopolysaccharide (LPS) induced HPC differentiation into myofibroblasts in the malignant transformation of HPCs. LPS induced HPCs to differentiate into myofibroblasts, which led to the proliferation and malignant transformation of HPCs by secretion of IL- 6 and TNF- $\alpha$. The potential mechanism was that LPS/Toll-like receptor 4 (TLR4) signaling induced differentiation of HPCs into myofibroblasts and production of IL- 6 and TNF- $\alpha$. IL- 6 and TNF- $\alpha$ promoted the upregulation of epidermal growth factor receptor $(E G F R)$ and downregulation of phosphatase and tensin homolog $(P T E N)$ through long non-coding RNA (lncRNA) regulation, which resulted in the aberrant expression of Ras and p53 signaling pathways in HPCs and finally enhanced the proliferation and malignant transformation of HPCs. ns, not significant

clean reads were aligned to the rat genome, using UCSC Rat reference [build Rn4]. No more than five mismatches were allowed in the alignment. The gene expression level was calculated using the RPKM (Reads Per Kilobase Million) method [41]. Differentially expressed transcript of each biological replicate was identified using two-fold change ( $\log 2$ fold change $\geq 1$ or $\leq-1$ ), statistical test referring to the significance of digital gene expression profiles [42], and a 0.001 Benjamini and Hochberg false discovery rate (FDR) [43]-corrected $p$-value cut-off for the thresholds. Only those transcripts that were upregulated or downregulated in three biological replicates were identified as differentially expressed transcripts. RNA isolation, library construction, RNA-sequencing, reads alignment, and DET identification were performed at Wuxi AppTec, China.

\section{Real-time PCR}

Total RNA was extracted using Trizol reagent according to the manufacturer's protocol. Real-time PCR was performed in a total reaction volume of $10 \mu \mathrm{l}(5 \mu \mathrm{l} \mathrm{SYBR}$ green, $1 \mu \mathrm{l}$ forward and reverse specific primers, respectively, $2 \mu \mathrm{l}$ complementary DNA and $\left.1 \mu \mathrm{l} \mathrm{ddH}_{2} \mathrm{O}\right)$. PCR conditions used were: $95^{\circ} \mathrm{C}$ for $10 \mathrm{~min}$, followed by 40 cycles of $95^{\circ} \mathrm{C}$ for $15 \mathrm{~s}, 60^{\circ} \mathrm{C}$ for $30 \mathrm{~s}$, and $72{ }^{\circ} \mathrm{C}$ for $30 \mathrm{~s}$.

\section{FACS analysis}

In this analysis, $20 \mu \mathrm{l}$ of anti- $\alpha$-SMA antibody was added to $100 \mu \mathrm{l}$ of cell suspension $\left(1 \times 10^{6}\right.$ cells $\left./ \mathrm{ml}\right)$ and incubated at $4^{\circ} \mathrm{C}$ for $30 \mathrm{~min}$, and further stained with secondary
Alexa488-conjugated goat anti-rabbit IgG (Invitrogen, Carlsbad, CA, USA) antibody at $4{ }^{\circ} \mathrm{C}$ for $30 \mathrm{~min}$. Alexa 488 conjugated isotype IgG antibody was used as a negative control. The stained cells were analyzed on a FACS flow cytometer (Becton Dickinson, San Jose, CA, USA).

\section{Bio-Plex assay}

Concentrations of chronic inflammatory factors were measured by the multiplex technology (Millipore, Darmstadt, Germany), according to the manufacturer's protocol (BioRad, USA).

\section{Enzyme-linked immunosorbent assay}

CM was collected from LPS-disposed WB-F344 cells and centrifuged at $1000 \mathrm{rpm}$ for $5 \mathrm{~min}$ to obtain the supernatant. The levels of IL- 6 and TNF- $\alpha$ in CM were determined using Enzyme-Linked Immunosorbent Assay (ELISA) Kit (R\&D Systems, USA), according to the manufacturer's instructions. Serum levels of LPS in rats were measured by ELISA, using the Rat Endotoxin (ET) ELISA Assay Kit (H178, Jiancheng, Nanjing, China)

\section{Microarray analysis}

Microarray analysis was performed by KangChen Bio-tech (Shanghai, P.R. China). Expression profiles of lncRNA and mRNA were analyzed using a Rat LncRNA Array (Arraystar, Rockville, MD, USA). Data were analyzed using the Agilent Feature Extraction software. Further analysis was carried out using the GeneSpring GX v11.5.1 software package (Agilent Technologies).

\section{Correlation and co-expression analysis}

The co-expression analysis was based on calculating the Pearson's correlation coefficient (PCC) between coding genes and non-coding transcripts, according to their expression levels. The absolute value of parameter PCC $\geq 0.90, p$ value $<0.01$ and FDR $<0.01$ was recommended and retained for further analysis.

\section{Gene silencing mediated by lentivirus-delivered shRNA}

We designed three shRNA candidates with TLR4 target sequences. Scramble shRNA served as the negative control. pGCL-GFP lentiviral particles encoded GFP and shRNAs (Obio Technology, Shanghai, China). Gene expression, after lentiviral infection, was determined by western blot on the fourth day. The transfected cells, after 6-8 passages, were used for subsequent experiments. 


\section{Western blot}

Protein samples were collected and quivalent aliquots of protein were electrophoresed on a $10 \%$ sodium dodecyl sulfate/polyacrylamide gel, in $1 \times$ Tris-glycine buffer, followed by transfer to nitrocellulose membranes, and incubated with primary antibodies, overnight at $4{ }^{\circ} \mathrm{C}$. Thereafter, the nitrocellulose membranes were incubated with secondary antibody for $1 \mathrm{~h}$, at room temperature. The immunoreactive proteins were detected by enhanced chemiluminescence substrate and the blot was scanned, and densitometric analysis with the Image $\mathbf{J}$ software was performed. The primary antibodies used in our experiment included $\alpha$-SMA (Abcam, diluted 1:1000), TLR4 (Abcam, diluted 1:1000) and glyceraldehyde 3-phosphate dehydrogenase (Bioworld, diluted 1:5000).

\section{Statistical analysis}

All experiments were performed at least three times. Analysis of variance was performed using GraphPad Prism 5.0 (GraphPad Software). Quantitative data were expressed as mean $\pm S D$, for each experiment. Significance between groups was performed using Student's $t$ test. Clinical data analysis was performed using SPSS 20.0 for Windows (SPSS Inc., Chicago, IL, USA); Pearson's correlation coefficient was used to determine correlations between continuous normally distributed variables. Kaplan-Meier analysis was used to determine the recurrence. Log-rank test was used to compare patients' recurrence time between each group. Statistical significances are indicated by $* p<0.05$, $* * p<0.01$, and $* * * p<0.001$.

Acknowledgements This project was supported by the National Key R\&D Program of China (Grant No. 2018YFA0107502); National Natural Science Foundation of China (Grant Nos. 81772940, 81702320, 81630070, 81673641, 81572444, 31700788, 81872243, 81802737); Special Funds for National Key Sci-Tech Special Project of China (Grant No. 2018ZX10723204-005-004); and Shanghai Science and Technology Committee (Grant Nos. 16ZR1400200, 16JC1405200, 16YF1415000); Science Fund for Creative Research Groups, NSFC, China (Grant No. 81521091).

Author contributions L-xW and Z-pH were responsible for the overall concept, design, and supervision of the study. W-tL, Y-yJ, and LG performed the experiments and data analysis, as well as manuscript writing. RL, XY, Q-dZ, and YY performed the experiments. X-rP, X$\mathrm{jH}$, and $\mathrm{YM}$ analyzed the data.

\section{Compliance with ethical standards}

Conflict of interest The authors declare that they have no conflict of interest.

Publisher's note: Springer Nature remains neutral with regard to jurisdictional claims in published maps and institutional affiliations.

\section{References}

1. El-Serag HB. Hepatocellular carcinoma. $\mathrm{N}$ Engl $\mathrm{J}$ Med. 2011;365:1118-1127.

2. Weber A, Boege Y, Reisinger F, Heikenwalder M. Chronic liver inflammation and hepatocellular carcinoma: persistence matters. Swiss Med Wkly. 2011;141:w13197.

3. Zhang DY, Friedman SL. Fibrosis-dependent mechanisms of hepatocarcinogenesis. Hepatology. 2012;56:769-75.

4. Michalopoulos GK. Liver regeneration: alternative epithelial pathways. Int J Biochem Cell Biol. 2011;43:173-79.

5. Turner R, et al. Human hepatic stem cell and maturational liver lineage biology. Hepatology. 2011;53:1035-45.

6. Weng HL, et al. IFN-gamma inhibits liver progenitor cell proliferation in $\mathrm{HBV}$-infected patients and in 3,5-diethoxycarbonyl1,4-dihydrocollidine diet-fed mice. J Hepatol. 2013;59:738-45.

7. Strain AJ, Crosby HA. Hepatic stem cells. Gut. 2000;46:743-45.

8. Clouston AD, et al. Fibrosis correlates with a ductular reaction in hepatitis C: roles of impaired replication, progenitor cells and steatosis. Hepatology. 2005;41:809-18.

9. Lukacs-Kornek V, Lammert F. The progenitor cell dilemma: cellular and functional heterogeneity in assistance or escalation of liver injury. J Hepatol. 2017;66:619-30.

10. Chobert MN, et al. Liver precursor cells increase hepatic fibrosis induced by chronic carbon tetrachloride intoxication in rats. Lab Invest. 2012;92:135-50.

11. Sekiya S, Miura S, Matsuda-Ito K, Suzuki A. Myofibroblasts derived from hepatic progenitor cells create the tumor microenvironment. Stem Cell Rep. 2016;7:1130-39.

12. Pan XR, et al. Lipopolysaccharide induces the differentiation of hepatic progenitor cells into myofibroblasts via activation of the Hedgehog signaling pathway. Cell Cycle. 2017;16:1357-65.

13. Arimilli S, Johnson JB, Alexander-Miller MA, Parks GD. TLR-4 and -6 agonists reverse apoptosis and promote maturation of simian virus 5-infected human dendritic cells through NFkBdependent pathways. Virology. 2007;365:144-56.

14. Ha T, et al. Lipopolysaccharide-induced myocardial protection against ischaemia/reperfusion injury is mediated through a PI3K/ Akt-dependent mechanism. Cardiovasc Res. 2008;78:546-53.

15. Darnaud M, Faivre J, Moniaux N. Targeting gut flora to prevent progression of hepatocellular carcinoma. J Hepatol. 2013;58:385-7.

16. Dapito DH, et al. Promotion of hepatocellular carcinoma by the intestinal microbiota and TLR4. Cancer Cell. 2012;21:504-16.

17. Cirera I, et al. Bacterial translocation of enteric organisms in patients with cirrhosis. J Hepatol. 2001;34:32-7.

18. Li XY, et al. Lipopolysaccharide promotes tumorigenicity of hepatic progenitor cells by promoting proliferation and blocking normal differentiation. Cancer Lett. 2017;386:35-46.

19. Libbrecht L, et al. Hepatic progenitor cells in hepatocellular adenomas. Am J Surg Pathol. 2001;25:1388-96.

20. Hruban RH, Maitra A, Kern SE, Goggins M. Precursors to pancreatic cancer. Gastroenterol Clin N Am. 2007;36:831-49.

21. Li W, et al. STK4 regulates TLR pathways and protects against chronic inflammation-related hepatocellular carcinoma. J Clin Invest. 2015;125:4239-54.

22. Libbrecht L, Desmet V, Van Damme B, Roskams T. Deep intralobular extension of human hepatic 'progenitor cells' correlates with parenchymal inflammation in chronic viral hepatitis: can 'progenitor cells' migrate? J Pathol. 2000;192:373-8.

23. Lowes KN, Brennan BA, Yeoh GC, Olynyk JK. Oval cell numbers in human chronic liver diseases are directly related to disease severity. Am J Pathol. 1999;154:537-41.

24. Katoonizadeh A, Nevens F, Verslype C, Pirenne J, Roskams T. Liver regeneration in acute severe liver impairment: a clinicopathological correlation study. Liver Int. 2006;26:1225-33. 
25. Falkowski O, et al. Regeneration of hepatocyte 'buds' in cirrhosis from intrabiliary stem cells. J Hepatol. 2003;39:357-64.

26. Harmey $\mathrm{JH}$, et al. Lipopolysaccharide-induced metastatic growth is associated with increased angiogenesis, vascular permeability and tumor cell invasion. Int J cancer. 2002;101:415-22.

27. Hamesch K, Borkham-Kamphorst E, Strnad P, Weiskirchen R. Lipopolysaccharide-induced inflammatory liver injury in mice. Lab Anim. 2015;49(1 Suppl.):37-46.

28. Jing YY, et al. Toll-like receptor 4 signaling promotes epithelialmesenchymal transition in human hepatocellular carcinoma induced by lipopolysaccharide. BMC Med. 2012;10:98.

29. Lai FB, et al. Lipopolysaccharide supports maintaining the stemness of CD133(+) hepatoma cells through activation of the NF-kappaB/HIF-1alpha pathway. Cancer Lett. 2016;378:131-41.

30. $\mathrm{He} \mathrm{G}$, et al. Identification of liver cancer progenitors whose malignant progression depends on autocrine IL-6 signaling. Cell. 2013;155:384-96.

31. Park EJ, et al. Dietary and genetic obesity promote liver inflammation and tumorigenesis by enhancing IL- 6 and TNF expression. Cell. 2010;140:197-208.

32. Yang $\mathrm{L}$, et al. Transforming growth factor-beta signaling in hepatocytes promotes hepatic fibrosis and carcinogenesis in mice with hepatocyte-specific deletion of TAK1. Gastroenterology. 2013;144:1042-1054 e1044.

33. Ji J, et al. MicroRNA expression, survival, and response to interferon in liver cancer. New Engl J Med. 2009;361:1437-47.

34. Lowes KN, Croager EJ, Olynyk JK, Abraham LJ, Yeoh GC. Oval cell-mediated liver regeneration: role of cytokines and growth factors. J Gastroenterol Hepatol. 2003;18:4-12.
35. Zhen L, et al. Curcumin inhibits oral squamous cell carcinoma proliferation and invasion via EGFR signaling pathways. Int $\mathbf{J}$ Clin Exp Pathol. 2014;7:6438-46.

36. Vinciguerra $M$, et al. Unsaturated fatty acids promote hepatoma proliferation and progression through downregulation of the tumor suppressor PTEN. J Hepatol. 2009;50: $1132-41$.

37. Wang Y, et al. The long noncoding RNA lncTCF7 promotes selfrenewal of human liver cancer stem cells through activation of Wnt signaling. Cell Stem Cell. 2015;16:413-25.

38. Yuan $\mathrm{JH}$, et al. A long noncoding RNA activated by TGF-beta promotes the invasion-metastasis cascade in hepatocellular carcinoma. Cancer Cell. 2014;25:666-81.

39. Uthaya Kumar DB, et al. TLR4 signaling via NANOG cooperates with STAT3 to activate Twist1 and promote formation of tumorinitiating stem-like cells in livers of mice. Gastroenterology. 2016;150:707-19.

40. Zhu XD, et al. High expression of macrophage colony-stimulating factor in peritumoral liver tissue is associated with poor survival after curative resection of hepatocellular carcinoma. J Clin Oncol. 2008;26:2707-16.

41. Mortazavi A, Williams BA, McCue K, Schaeffer L, Wold B. Mapping and quantifying mammalian transcriptomes by RNASeq. Nat Methods. 2008;5:621-28.

42. Audic S, Claverie JM. The significance of digital gene expression profiles. Genome Res. 1997;7:986-95.

43. Benjamini YHY. Controlling the false discovery rate: a practical and powerful approach to multiple testing. J R Stat Soc B. 1995;57:289-300. 